\title{
Rigidification of quasi-categories
}

\author{
DANIEL DUGGER \\ DAVID I SPIVAK
}

\begin{abstract}
We give a new construction for rigidifying a quasi-category into a simplicial category, and prove that it is weakly equivalent to the rigidification given by Lurie. Our construction comes from the use of necklaces, which are simplicial sets obtained by stringing simplices together. As an application of these methods, we use our model to reprove some basic facts from Lurie [13] about the rigidification process.
\end{abstract}

55U40; 18G30, 18B99

\section{Introduction}

Quasi-categories are a certain generalization of categories, where in addition to the usual 1-morphisms one has $n$-morphisms for every natural number $n$. They are special among higher categories in that they have the property that for $n>1$ the $n$-morphisms are all invertible in an appropriate sense. Quasi-categories have been extensively studied by Cordier and Porter [6], by Joyal [11; 12], and by Lurie [13]. If $K$ is a quasi-category and $x$ and $y$ are two objects of $K$, then one may associate a "mapping space" $K(x, y)$ which is a simplicial set. There are many different constructions for these mapping spaces, but in [13] one particular model is given for which there are composition maps $K(y, z) \times K(x, y) \rightarrow K(x, z)$ giving rise to a simplicial category. This simplicial category is denoted $\mathfrak{C}(K)$, and it may be thought of as a rigidification of the quasicategory $K$. It is proven in [13] that the homotopy theories of quasi-categories and simplicial categories are equivalent via this functor.

In this paper we introduce some new models for the mapping spaces $K(x, y)$, which are particularly easy to describe and particularly easy to use-in fact they are just the nerves of ordinary categories (ie, 1-categories). Like Lurie's model, our models admit composition maps giving rise to a simplicial category; so we are giving a new method for rigidifying quasi-categories. We prove that our construction is homotopy equivalent (as a simplicial category) to Lurie's $\mathfrak{C}(K)$. Moreover, because our mapping spaces are nerves of categories there are many standard tools available for analyzing their homotopy types. We demonstrate the effectiveness of this by giving new proofs of some basic facts about the functor $\mathfrak{C}(-)$. 
One payoff of this approach is that it is possible to give a streamlined proof of Lurie's Quillen equivalence between the homotopy theory of quasi-categories and simplicial categories. This requires, however, a more detailed study of the model category structure on quasi-categories. We will take this up in a sequel [8] and prove the Quillen equivalence there.

\subsection{Mapping spaces via simplicial categories}

Now we describe our results in more detail. A quasi-category is a simplicial set that has the right-lifting-property with respect to inner horn inclusions $\Lambda_{i}^{n} \rightarrow \Delta^{n}, 0<i<n$. It turns out that there is a unique model structure on $s$ et where the cofibrations are the monomorphisms and the fibrant objects are the quasi-categories; this will be called the Joyal model structure and denoted $s \mathscr{S e t}_{J}$. The weak equivalences in $s \mathscr{S e t}_{J}$ will here be called Joyal equivalences (they are called "weak categorical equivalences" in [12]). The existence of the Joyal model structure will not be needed in this paper, although it provides some useful context. The notions of quasi-categories and Joyal equivalences, however, will be used in several places. See Section 2.3 for additional background.

There is a functor, constructed in [13], which sends any simplicial set $K$ to a corresponding simplicial category $\mathfrak{C}(K) \in s^{\mathscr{C}}$ at. This is the left adjoint in a Quillen pair

$$
\mathfrak{C}: s \mathscr{S e t}_{J} \rightleftarrows s b_{\text {at }}: N,
$$

where $N$ is called the coherent nerve. To avoid later confusion, we note that if a simplicial category $\mathscr{D}$ has discrete mapping spaces - that is, if it is just an ordinary category-then $N \mathscr{D}$ coincides with the classical nerve construction. The functor $N$ can be described quite explicitly (see Section 2), but the functor $\mathfrak{C}$ is in comparison a little mysterious. In [13] each $\mathfrak{C}(K)$ is defined as a certain colimit in the category $s \mathscr{C}$ at, but colimits in $s \mathscr{C}$ at are notoriously difficult to understand.

Our main goal in this paper is to give a different model for the functor $\mathfrak{C}$. Define a necklace (which we picture as "unfastened") to be a simplicial set of the form

$$
\Delta^{n_{0}} \vee \Delta^{n_{1}} \vee \cdots \vee \Delta^{n_{k}}
$$

where each $n_{i} \geq 0$ and where in each wedge the final vertex of $\Delta^{n_{i}}$ has been glued to the initial vertex of $\Delta^{n_{i+1}}$. Necklaces were used by Baues in his study of loop spaces [2].

The first and last vertex in any necklace $T$ are denoted $\alpha_{T}$ and $\omega_{T}$, respectively (or just $\alpha$ and $\omega$ if $T$ is obvious from context). If $S$ and $T$ are two necklaces, then by $S \vee T$ we mean the necklace obtained in the evident way, by gluing the final vertex $\omega_{S}$ 
of $S$ to the initial vertex $\alpha_{T}$ of $T$. Write $\mathcal{N e c}$ for the category whose objects are necklaces and where a morphism is a map of simplicial sets which preserves the initial and final vertices.

Let $S \in s \mathscr{S e t}$ and let $a, b \in S_{0}$. If $T$ is a necklace, we use the notation

$$
T \rightarrow S_{a, b}
$$

to indicate a morphism of simplicial sets $T \rightarrow S$ which sends $\alpha_{T}$ to $a$ and $\omega_{T}$ to $b$. Let $\left(\mathcal{N} e c \downarrow S_{a, b}\right)$ denote the evident category whose objects are pairs $\left[T, T \rightarrow S_{a, b}\right]$ where $T$ is a necklace. Note that for $a, b, c \in S_{0}$ there is a functor

$$
\left(\mathcal{N} e c \downarrow S_{b, c}\right) \times\left(\mathcal{N} e c \downarrow S_{a, b}\right) \longrightarrow\left(\mathcal{N} e c \downarrow S_{a, c}\right)
$$

which sends the pair $\left(\left[T_{2}, T_{2} \rightarrow S_{b, c}\right],\left[T_{1}, T_{1} \rightarrow S_{a, b}\right]\right)$ to $\left[T_{1} \vee T_{2}, T_{1} \vee T_{2} \rightarrow S_{a, c}\right]$. Let $\mathfrak{C}^{\text {nec }}(S)$ be the function assigning to any $a, b \in S_{0}$ the simplicial set $\mathfrak{C}^{\text {nec }}(S)(a, b)=$ $N\left(\mathcal{N} e c \downarrow S_{a, b}\right)$ (the classical nerve of the 1-category $\left(\mathcal{N} e c \downarrow S_{a, b}\right)$ ). The above pairings of categories induce pairings on the nerves, which makes $\mathfrak{C}^{\text {nec }}(S)$ into a simplicial category with object set $S_{0}$.

1.3 Theorem There is a natural zigzag of weak equivalences of simplicial categories between $\mathfrak{C}^{\text {nec }}(S)$ and $\mathfrak{C}(S)$, for all simplicial sets $S$.

In the above result, the weak equivalences for simplicial categories are the so-called "DK-equivalences" used by Bergner in [3] and first defined by Dwyer and Kan [9]. See Section 2 for this notion.

In this paper we also give an explicit description of the mapping spaces in the simplicial category $\mathfrak{C}(S)$. A rough statement is given below, but see Section 4 for more details.

1.4 Theorem Let $S$ be a simplicial set and let $a, b \in S$. Then the mapping space $X=\mathfrak{C}(S)(a, b)$ is the simplicial set whose $n$-simplices are triples subject to a certain equivalence relations. The triples consist of a necklace $T$, a map $T \rightarrow S_{a, b}$, and a flag $\vec{T}=\left\{T^{0} \subseteq \cdots \subseteq T^{n}\right\}$ of vertices in $T$. For the equivalence relation, see Corollary 4.4. The face maps and degeneracy maps are obtained by removing or repeating elements $T^{i}$ in the flag.

The pairing

$$
\mathfrak{C}(S)(b, c) \times \mathfrak{C}(S)(a, b) \longrightarrow \mathfrak{C}(S)(a, c)
$$

sends the pair of $n$-simplices $\left(\left[T \rightarrow S ; \overrightarrow{T^{i}}\right],\left[U \rightarrow S, \overrightarrow{U^{i}}\right]\right)$ to $\left[U \vee T \rightarrow S, \overrightarrow{U^{i} \cup T^{i}}\right]$. 
Theorem 1.3 turns out to be very useful in the study of the functor $\mathfrak{C}$. There are many tools in classical homotopy theory for understanding the homotopy types of nerves of 1-categories, and via Theorem 1.3 these tools can be applied to understand mapping spaces in $\mathfrak{C}(S)$. We demonstrate this technique in Section 6 by proving, in a new way, the following two properties of $\mathfrak{C}$ found in [13].

1.5 Theorem Let $X$ and $Y$ be simplicial sets.

(a) The natural map $\mathfrak{C}(X \times Y) \rightarrow \mathfrak{C}(X) \times \mathfrak{C}(Y)$ is a weak equivalence of simplicial categories.

(b) If $X \rightarrow Y$ is a Joyal equivalence then $\mathfrak{C}(X) \rightarrow \mathfrak{C}(Y)$ is a weak equivalence.

\subsection{Notation and terminology}

We will sometimes use $s \mathscr{S e t}_{K}$ to refer to the usual model structure on simplicial sets, which we'll term the Kan model structure. The fibrations are the Kan fibrations, the weak equivalences (called Kan equivalences from now on) are the maps which induce homotopy equivalences on geometric realizations, and the cofibrations are the monomorphisms.

We will often be working with the category $s \mathscr{S e t}_{*, *}=\left(\partial \Delta^{1} \downarrow s \mathscr{S e t}_{e t}\right.$. Note that $\mathcal{N e c}$ is a full subcategory of $s \mathscr{S e t}_{*, *}$.

An object of $s \mathscr{S e t}_{*, *}$ is a simplicial set $X$ with two distinguished points $a$ and $b$. We sometimes (but not always) write $X_{a, b}$ for $X$, to remind us that things are taking place in $s \mathscr{S e t}_{*, *}$ instead of $s \mathscr{P e t}$.

If $\mathscr{C}$ is a (simplicial) category containing objects $X$ and $Y$, we write $\mathscr{C}(X, Y)$ for the (simplicial) set of morphisms from $X$ to $Y$.

Acknowledgements The first author was supported by NSF grant DMS-0604354. The second author was supported by ONR grant N000140910466. We are grateful to Emily Riehl for comments on an earlier version of this paper.

\section{Background on quasi-categories}

In this section we give the background on quasi-categories and simplicial categories needed in the rest of the paper. 


\subsection{Simplicial categories}

A simplicial category is a category enriched over simplicial sets; it can also be thought of as a simplicial object of $\mathscr{C}$ at in which the simplicial operators are all equal to the identity on the object sets. We use $s \mathscr{C}$ at to denote the category of simplicial categories. Given a simplicial category $\mathscr{D}$, let $\pi_{0} \mathscr{D}$ denote the ordinary category having the same object set, and where the set of morphisms from $x$ to $y$ is $\pi_{0}(\mathscr{D}(x, y))$; as this construction is functorial in $\mathscr{D}$, we regard $\pi_{0}: s \mathscr{b} a t \rightarrow \mathscr{C} a t$ as a functor.

A cofibrantly-generated model structure on $s \mathscr{C}$ at was developed in [3]. In this structure a map of simplicial categories $F: \mathscr{C} \rightarrow \mathscr{D}$ is a weak equivalence (sometimes called a DK-equivalence) if

(1) For all $a, b \in \mathrm{ob} \mathscr{C}$, the map $\mathscr{C}(a, b) \rightarrow \mathscr{D}(F a, F b)$ is a Kan equivalence of simplicial sets;

(2) The induced functor of ordinary categories $\pi_{0} F: \pi_{0} \mathscr{b} \rightarrow \pi_{0} \mathscr{D}$ is surjective on isomorphism classes of objects.

Likewise, the map $F$ is a fibration if

(1) For all $a, b \in \mathrm{ob} \mathscr{C}$, the map $\mathscr{C}(a, b) \rightarrow \mathscr{D}(F a, F b)$ is a Kan fibration of simplicial sets;

(2) For all $a \in \mathrm{ob} \mathscr{C}$ and $b \in \mathrm{ob} \mathscr{D}$, if $e: F a \rightarrow b$ is a map in $\mathscr{D}$ which becomes an isomorphism in $\pi_{0} \mathscr{D}$, then there is an object $b^{\prime} \in \mathscr{C}$ and a map $e^{\prime}: a \rightarrow b^{\prime}$ such that $F\left(e^{\prime}\right)=e$ and $e^{\prime}$ becomes an isomorphism in $\pi_{0} \mathscr{b}$.

The cofibrations are the maps which have the left lifting property with respect to the acyclic fibrations.

2.2 Remark The second part of the fibration condition seems a little awkward at first. In this paper we will actually have no need to think about fibrations of simplicial categories, but have included the definition for completeness.

Bergner writes down sets of generating cofibrations and acyclic cofibrations in [3].

\subsection{Quasi-categories and Joyal equivalences}

As mentioned in the introduction, there is a unique model structure on $s \mathscr{S}$ et with the properties that

(i) The cofibrations are the monomorphisms;

(ii) The fibrant objects are the quasi-categories. 
It is easy to see that there is at most one such structure. To do this, for any set $S$ let $\mathscr{E}(S)$ be the groupoid with object set $S$ having the property that for any two objects $s_{1}, s_{2} \in S$ there is a unique map $s_{1} \rightarrow s_{2}$. Let $E^{1}$ be the nerve of the groupoid $\mathscr{E}(\{0,1\})$. The $k$-simplices of $E^{1}$ may be identified with $(k+1)$-tuples consisting of 0 's and 1 's, where the face and degeneracy maps are the usual deletion and repetition of entries. Note that the geometric realization of $E^{1}$ is essentially the standard model for $S^{\infty}$. One may also describe $E^{1}$ as the 0 -coskeleton-see Artin and Mazur [1], for instance-of the set $\{0,1\}$. If $X$ is any simplicial set then maps $X \rightarrow E^{1}$ are in bijective correspondence with set maps $X_{0} \rightarrow\{0,1\}$.

Assume that a model structure on $s$ et exists having properties (i) and (ii) above. The map $E^{1} \rightarrow *$ has the right lifting property with respect to all monomorphisms, and so it will be an acyclic fibration in this structure. Therefore $X \times E^{1} \rightarrow X$ is also an acyclic fibration for any $X$, and hence $X \times E^{1}$ will be a cylinder object for $X$. Since every object is cofibrant, a map $A \rightarrow B$ will be a weak equivalence if and only if it induces bijections $[B, Z]_{E^{1}} \rightarrow[A, Z]_{E^{1}}$ for every quasi-category $Z$, where $[A, Z]_{E^{1}}$ means the coequalizer of $s \operatorname{Set}\left(A \times E^{1}, Z\right) \rightrightarrows s \operatorname{Set}(A, Z)$. Therefore the weak equivalences are determined by properties (i)-(ii), and since the cofibrations and weak equivalences are determined so are the fibrations. Thus, such a model category structure will be unique.

Motivated by the above discussion, we define a map of simplicial sets $A \rightarrow B$ to be a Joyal equivalence if it induces bijections $[B, Z]_{E^{1}} \rightarrow[A, Z]_{E^{1}}$ for every quasicategory $Z$.

That there actually exists a model structure satisfying (i) and (ii) is not so clear, but it was established by Joyal. See Joyal [11; 12], Lurie [13] for another proof (particularly Theorems 2.2.5.1 and 2.4.6.1), or the appendix of the sequel [8] for a compact presentation of Joyal's arguments. We will call this the Joyal model structure and denote it by $s \mathscr{S e t}_{J}$. The weak equivalences are defined differently in both [12] and [13], but of course turn out to be equivalent to the definition we have adopted here.

In the rest of the paper we will never use the Joyal model structure, only the notion of Joyal equivalence.

\subsection{Background on $\mathfrak{C}$ and $N$}

Given a simplicial category $S$, one can construct a simplicial set called the coherent nerve of $S$; see Cordier [5] and Lurie [13, 1.1.5]. We will now describe this construction.

Recall the adjoint functors $F: \mathscr{G r p h} \rightleftarrows \mathscr{C a t}: U$. Here $\mathscr{C} a t$ is the category of (small) 1-categories, and $\mathscr{G}_{r p h}$ is the category of directed graphs: a graph consists of a vertex 
set $X_{0}$, an edge set $X_{1}$, and two maps $X_{1} \rightrightarrows X_{0}$. The functor $U$ is the evident forgetful functor that takes a category $C$ to $\operatorname{Mor}(C) \rightrightarrows \mathrm{ob}(C)$, and $F$ is a free functor that adds in formal compositions. See Mac Lane [14, Chapter II.7].

Given any category $\mathscr{C}$ we may then consider the comonad resolution $(F U) \bullet(\mathscr{C})$ given by $[n] \mapsto(F U)^{n+1}(\mathscr{C})$. This is a simplicial category. There is a functor of simplicial categories $(F U) \bullet(\mathscr{C}) \rightarrow \mathscr{C}$ (where the latter is considered a discrete simplicial category). This functor induces a weak equivalence on all mapping spaces, a fact which can be seen by applying $U$, at which point the comonad resolution picks up a contracting homotopy. Note that this means that the simplicial mapping spaces in $(F U) \bullet(\mathscr{C})$ are all homotopy discrete.

Recall that $[n]$ denotes the category $0 \rightarrow 1 \rightarrow \cdots \rightarrow n$, where there is a unique map from $i$ to $j$ whenever $i \leq j$. We let $\mathfrak{C}\left(\Delta^{n}\right)$ denote the simplicial category $(F U)_{\bullet}([n])$. The mapping spaces in this simplicial category can be analyzed completely, and are as follows. For each $i$ and $j$, let $P_{i, j}$ denote the poset of all subsets of $\{i, i+1, \ldots, j\}$ containing $i$ and $j$ (ordered by inclusion). Note that the nerve of $P_{i, j}$ is isomorphic to the cube $\left(\Delta^{1}\right)^{j-i-1}$ if $j>i, \Delta^{0}$ if $j=i$, and the empty set if $j<i$. The nerves of the $P_{i, j}$ 's naturally form the mapping spaces of a simplicial category with object set $\{0,1, \ldots, n\}$, using the pairings $P_{j, k} \times P_{i, j} \rightarrow P_{i, k}$ given by union of sets.

2.5 Lemma There is an isomorphism of simplicial categories $\mathfrak{C}\left(\Delta^{n}\right) \cong N P$.

2.6 Remark The proof of the above lemma is a bit of an aside from the main thrust of the paper, so it is given in Appendix A. In fact we could have defined $\mathfrak{C}\left(\Delta^{n}\right)$ to be $N P$, which is what Lurie does in [13], and avoided the lemma entirely; the construction $(F U)_{\bullet}([n])$ will never again be used in this paper. Nevertheless, the identification of $N P$ with $(F U) \bullet([n])$ demonstrates that $N P$ arises naturally in this setting.

For any simplicial category $\mathscr{D}$, the coherent nerve of $\mathscr{D}$ is the simplicial set $N \mathscr{D}$ given by

$$
[n] \mapsto s \mathscr{C a t}\left(\mathfrak{C}\left(\Delta^{n}\right), \mathscr{D}\right) .
$$

It was proven by Lurie [13] that if every mapping space in $\mathscr{D}$ is a Kan complex, then $N \mathscr{D}$ is a quasi-category; see also Lemma 6.5 below.

The functor $N$ has a left adjoint. If we temporarily denote this by $\mathfrak{C}^{\prime}$, note that there are canonical bijections

$$
s^{\mathscr{C a t}}\left(\mathfrak{C}^{\prime}\left(\Delta^{n}\right), \mathscr{D}\right) \cong s \mathscr{S e t}\left(\Delta^{n}, N \mathscr{D}\right)=s \mathscr{C a t}\left(\mathfrak{C}\left(\Delta^{n}\right), \mathscr{D}\right) .
$$

It follows from this that $\mathfrak{C}^{\prime}\left(\Delta^{n}\right) \cong \mathfrak{C}\left(\Delta^{n}\right)$. It therefore makes sense to denote the left adjoint of $N$ as just $\mathfrak{C}: s \mathscr{S e t} \rightarrow s^{\mathscr{C}}$ at . 
Any simplicial set $K$ may be written as a colimit of simplices via the formula

$$
K \cong \operatorname{colim}_{\Delta^{n} \rightarrow K} \Delta^{n},
$$

and consequently one has

$$
\mathfrak{C}(K) \cong \operatorname{colim}_{\Delta^{n} \rightarrow K} \mathfrak{C}\left(\Delta^{n}\right)
$$

where the colimit takes place in $s$ bat. This formula is a bit unwieldy, however, in the sense that it does not give much concrete information about the mapping spaces in $\mathfrak{C}(K)$. The point of the next three sections is to obtain such concrete information, via the use of necklaces.

\section{Necklaces}

A necklace is a simplicial set obtained by stringing simplices together in succession. In this section we establish some basic facts about them, as well as facts about the more general category of ordered simplicial sets. When $T$ is a necklace we are able to give a complete description of the mapping spaces in $\mathfrak{C}(T)$ as nerves of certain posets, generalizing what was said for $\mathfrak{C}\left(\Delta^{n}\right)$ in the last section. See Proposition 3.8.

As briefly discussed in the introduction, a necklace is defined to be a simplicial set of the form

$$
\Delta^{n_{0}} \vee \Delta^{n_{1}} \vee \cdots \vee \Delta^{n_{k}}
$$

where each $n_{i} \geq 0$ and where in each wedge the final vertex of $\Delta^{n_{i}}$ has been glued to the initial vertex of $\Delta^{n_{i+1}}$. We say that the necklace is in preferred form if either $k=0$ or each $n_{i} \geq 1$.

Let $T=\Delta^{n_{0}} \vee \Delta^{n_{1}} \vee \cdots \vee \Delta^{n_{k}}$ be in preferred form. Each $\Delta^{n_{i}}$ is called a bead of the necklace. A joint of the necklace is either an initial or a final vertex in some bead. Thus, every necklace has at least one vertex, one bead, and one joint; $\Delta^{0}$ is not a bead in any necklace except in the necklace $\Delta^{0}$ itself.

Given a necklace $T$, write $V_{T}$ and $J_{T}$ for the sets of vertices and joints of $T$. Note that $V_{T}=T_{0}$ and $J_{T} \subseteq V_{T}$. Both $V_{T}$ and $J_{T}$ are totally ordered, by saying $a \leq b$ if there is a directed path in $T$ from $a$ to $b$. The initial and final vertices of $T$ are denoted $\alpha_{T}$ and $\omega_{T}$ (and we sometimes drop the subscript); note that $\alpha_{T}, \omega_{T} \in J_{T}$.

Every necklace $T$ comes with a particular map $\partial \Delta^{1} \rightarrow T$ which sends 0 to the initial vertex of the necklace, and 1 to the final vertex. If $S$ and $T$ are two necklaces, then by $S \vee T$ we mean the necklace obtained in the evident way, by gluing the final vertex of $S$ to the initial vertex of $T$. Let $\mathcal{N} e c$ denote the full subcategory of 
${ }_{s}$ et $_{*, *}=\left(\partial \Delta^{1} \downarrow s \varphi e t\right)$ whose objects are necklaces $\partial \Delta^{1} \rightarrow T$. By a map of necklaces we mean a map in this category. We will sometimes talk about $\mathcal{N e c}$ as though it is a subcategory of $s \mathscr{S}$ et.

A simplex is a necklace with one bead. A spine is a necklace in which every bead is a $\Delta^{1}$. Every necklace $T$ has an associated simplex and spine, which we now define. Let $\Delta[T]$ be the simplex whose vertex set is the same as the (ordered) vertex set of $T$. Likewise, let $\operatorname{Spi}[T]$ be the longest spine inside of $T$. Note that there are inclusions $\operatorname{Spi}[T] \hookrightarrow T \hookrightarrow \Delta[T]$. The assignment $T \rightarrow \Delta[T]$ is a functor, but $T \rightarrow \operatorname{Spi}[T]$ is not (for instance, the unique map of necklaces $\Delta^{1} \rightarrow \Delta^{2}$ does not induce a map on spines).

\subsection{Ordered simplicial sets}

If $T \rightarrow T^{\prime}$ is a map of necklaces, then the image of $T$ is also a necklace. To prove this, as well as for several other reasons scattered throughout the paper, it turns out to be very convenient to work in somewhat greater generality.

If $X$ is a simplicial set, define a relation on its 0 -simplices by saying that $x \preceq y$ if there exists a spine $T$ and a map $T \rightarrow X$ sending $\alpha_{T} \mapsto x$ and $\omega_{T} \mapsto y$. In other words, $x \preceq y$ if there is a directed path from $x$ to $y$ inside of $X$. Note that this relation is clearly reflexive and transitive, but not necessarily antisymmetric: that is, if $x \preceq y$ and $y \preceq x$ it need not be true that $x=y$. In cases where we are considering different simplicial sets $X$ and $Y$, we will write $\preceq_{X}$ and $\preceq_{Y}$ to distinguish the relations.

\subsection{Definition A simplicial set $X$ is ordered if}

(i) The relation $\preceq$ defined on $X_{0}$ is antisymmetric;

(ii) A simplex $x \in X_{n}$ is determined by its sequence of vertices $x(0) \preceq \cdots \preceq x(n)$; ie no two distinct $n$-simplices have identical vertex sequences.

Note the role of degenerate simplices in condition (ii). For example, notice that $\Delta^{1} / \partial \Delta^{1}$ is not an ordered simplicial set.

3.3 Lemma Let $X$ and $Y$ denote ordered simplicial sets and let $f: X \rightarrow Y$ be a map.

(1) The category of ordered simplicial sets is closed under taking finite limits.

(2) Every necklace is an ordered simplicial set.

(3) If $X^{\prime} \subseteq X$ is a simplicial subset, then $X^{\prime}$ is also ordered. 
(4) The map $f$ is completely determined by the map $f_{0}: X_{0} \rightarrow Y_{0}$ on vertices.

(5) If $f_{0}$ is injective then so is $f$.

(6) The image of an n-simplex $x: \Delta^{n} \rightarrow X$ is of the form $\Delta^{k} \hookrightarrow X$ for some $k \leq n$.

(7) If $T$ is a necklace and $y: T \rightarrow X$ is a map, then its image is a necklace.

Proof For (1), the terminal object is a point with its unique ordering. Given a diagram of the form

$$
X \longrightarrow Z \longleftarrow Y
$$

let $A=X \times_{Z} Y$. It is clear that if $(x, y) \preceq_{A}\left(x^{\prime}, y^{\prime}\right)$ then both $x \preceq_{X} x^{\prime}$ and $y \preceq_{Y} y^{\prime}$ hold, and so antisymmetry of $\preceq_{A}$ follows from that of $\preceq_{X}$ and $\preceq_{Y}$. Condition (ii) from Definition 3.2 is easy to check.

Parts (2)-(5) are easy, and left to the reader.

For (6), the sequence $x(0), \ldots, x(n) \in X_{0}$ may have duplicates; let $d: \Delta^{k} \rightarrow \Delta^{n}$ denote any face such that $x \circ d$ contains all vertices $x(j)$ and has no duplicates. Note that $x \circ d$ is an injection by (5). A certain degeneracy of $x \circ d$ has the same vertex sequence as $x$. Since $X$ is ordered, $x$ is this degeneracy of $x \circ d$. Hence, $x \circ d: \Delta^{k} \hookrightarrow X$ is the image of $x$.

Claim (7) follows from (6).

The following notion is also useful:

3.4 Definition Let $A$ and $X$ be ordered simplicial sets. A map $A \rightarrow X$ is called a simple inclusion if it has the right lifting property with respect to the canonical inclusions $\partial \Delta^{1} \hookrightarrow T$ for all necklaces $T$. (Note that such a map really is an inclusion using Lemma 3.3(5), because it has the lifting property for $\partial \Delta^{1} \rightarrow \Delta^{0}$ ).

It is sometimes useful to think of a map $T \rightarrow X$, where $T$ is a necklace, as being a "generalized path" in $X$. The notion of simple inclusion says if there is such a "generalized path" in $X$ whose endpoints are both in $A$, then it must lie entirely within $A$. As a simple exercise, the reader might check that four out of the five inclusions $\Delta^{1} \hookrightarrow \Delta^{1} \times \Delta^{1}$ are simple inclusions.

3.5 Lemma $A$ simple inclusion $A \hookrightarrow X$ of ordered simplicial sets has the right lifting property with respect to the maps $\partial \Delta^{k} \hookrightarrow \Delta^{k}$ for all $k \geq 1$. 
Proof Suppose the following square is given:

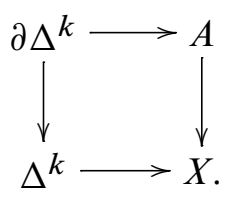

By restricting the map $\partial \Delta^{k} \rightarrow A$ to $\partial \Delta^{1} \hookrightarrow \partial \Delta^{k}$ (given by the initial and final vertices of $\partial \Delta^{k}$ ), we get a corresponding lifting square with $\partial \Delta^{1} \hookrightarrow \Delta^{k}$. Since $A \rightarrow X$ is a simple inclusion, this new square has a lift $l: \Delta^{k} \rightarrow A$. It is not immediately clear that $l$ restricted to $\partial \Delta^{k}$ equals our original map, but the two maps are equal after composing with $A \rightarrow X$; since $A \rightarrow X$ is a monomorphism, the two maps are themselves equal.

3.6 Lemma Suppose that $X \leftarrow A \rightarrow Y$ is a diagram of ordered simplicial sets, and both $A \rightarrow X$ and $A \rightarrow Y$ are simple inclusions. Then the pushout $B=X \amalg_{A} Y$ is an ordered simplicial set, and the inclusions $X \hookrightarrow B$ and $Y \hookrightarrow B$ are both simple.

Proof We first show that the maps $X \hookrightarrow B$ and $Y \hookrightarrow B$ have the right-lifting-property with respect to $\partial \Delta^{1} \hookrightarrow T$ for all necklaces $T$. To see this, suppose that $u, v \in X$ are vertices, $T$ is a necklace, and $f: T \rightarrow B_{u, v}$ is a map; we want to show that $f$ factors through $X$. Note that any simplex $\Delta^{k} \rightarrow B$ either factors through $X$ or through $Y$. Suppose that $f$ does not factor through $X$. From the set of beads of $T$ which do not factor through $X$, take any maximal subset $T^{\prime}$ in which all the beads are adjacent. Then we have a necklace $T^{\prime} \subseteq T$ such that $f\left(T^{\prime}\right) \subseteq Y$. If there exists a bead in $T$ prior to $\alpha_{T^{\prime}}$, then it must map into $X$ since $T^{\prime}$ was maximal; therefore $f\left(\alpha_{T^{\prime}}\right)$ would lie in $X \cap Y=A$. Likewise, if there is no bead prior to $\alpha_{T^{\prime}}$ then $f\left(\alpha_{T^{\prime}}\right)=u$ and so again $f\left(\alpha_{T^{\prime}}\right)$ lies in $X \cap Y=A$. Similar remarks apply to show that $f\left(\omega_{T^{\prime}}\right)$ lies in $A$. At this point the fact that $A \hookrightarrow Y$ is a simple inclusion implies that $f\left(T^{\prime}\right) \subseteq A \subseteq X$, which is a contradiction. So in fact $f$ factored through $X$.

We have shown that $X \hookrightarrow B$ (and dually $Y \hookrightarrow B$ ) has the right-lifting-property with respect to maps $\partial \Delta^{1} \hookrightarrow T$, for $T$ a necklace. Now we show that $B$ is ordered, and this will complete the proof. So suppose $u, v \in B$ are such that $u \preceq v$ and $v \preceq u$. There there are spines $T$ and $U$ and maps $T \rightarrow B_{u, v}, U \rightarrow B_{v, u}$. Consider the composite spine $T \vee U \rightarrow B_{u, u}$. If $u \in X$, then by the proven right-lifting-property for $X \hookrightarrow B$ it follows that the image of $T \vee U$ maps entirely into $X$; so $u \preceq_{X} v$ and $v \preceq_{X} u$, which means $u=v$ because $X$ is ordered. The same argument works if $u \in Y$, so this verifies antisymmetry of $\preceq_{B}$.

To verify condition (ii) of Definition 3.2, suppose $p, q: \Delta^{k} \rightarrow B$ are $k$-simplices with the same sequence of vertices; we wish to show $p=q$. We know that $p$ factors 
through $X$ or $Y$, and so does $q$; if both factor through $Y$, then the fact that $Y$ is ordered implies that $p=q$ (similarly for $X$ ). So we may assume $p$ factors through $X$ and $q$ factors through $Y$. By induction on $k$, the restrictions of $p$ and $q$ to any proper face of $\Delta^{k}$ are equal; therefore $\left.p\right|_{\partial \Delta^{k}}$ and $\left.q\right|_{\partial \Delta^{k}}$ are equal, hence they factor through $A$. By Lemma 3.5 applied to $A \hookrightarrow X$, the map $p$ factors through $A$. Therefore it also factors through $Y$, and now we are done because $q$ also factors through $Y$ and $Y$ is ordered.

\subsection{Rigidification of necklaces}

Let $T$ be a necklace. Our next goal is to give a complete description of the simplicial category $\mathfrak{C}(T)$. The object set of this category is precisely $T_{0}$.

For vertices $a, b \in T_{0}$, let $V_{T}(a, b)$ denote the set of vertices in $T$ between $a$ and $b$, inclusive (with respect to the relation $\preceq$ ). Let $J_{T}(a, b)$ denote the union of $\{a, b\}$ with the set of joints between $a$ and $b$. There is a unique subnecklace of $T$ with joints $J_{T}(a, b)$ and vertices $V_{T}(a, b)$; let $\widetilde{B}_{0}, \widetilde{B}_{1}, \ldots \widetilde{B}_{k}$ denote its beads. There are canonical inclusions of each $\widetilde{B}_{i}$ to $T$. Hence, there is a natural map

$\mathfrak{C}\left(\widetilde{B}_{k}\right)\left(j_{k}, b\right) \times \mathfrak{C}\left(\widetilde{B}_{k-1}\right)\left(j_{k-1}, j_{k}\right) \times \cdots \times \mathfrak{C}\left(\widetilde{B}_{1}\right)\left(j_{1}, j_{2}\right) \times \mathfrak{C}\left(\widetilde{B}_{0}\right)\left(a, j_{1}\right) \rightarrow \mathfrak{C}(T)(a, b)$ obtained by first including the $\widetilde{B}_{i}$ 's into $T$ and then using the composition in $\mathfrak{C}(T)$ (where $j_{i}$ and $j_{i+1}$ are the joints of $\widetilde{B}_{i}$ ). We will see that this map is an isomorphism. Note that each of the sets $\mathfrak{C}\left(\widetilde{B}_{i}\right)(-,-)$ has an easy description, as in Lemma 2.5; from this one may extrapolate a corresponding description for $\mathfrak{C}(T)(-,-)$, to be explained next.

Let $C_{T}(a, b)$ denote the poset whose elements are the subsets of $V_{T}(a, b)$ which contain $J_{T}(a, b)$, and whose ordering is inclusion. There is a pairing of categories

$$
C_{T}(b, c) \times C_{T}(a, b) \rightarrow C_{T}(a, c)
$$

given by union of subsets.

Applying the nerve functor, we obtain a simplicial category $N C_{T}$ with object set $T_{0}$. For $a, b \in T_{0}$, an $n$-simplex in $N C_{T}(a, b)$ can be seen as a flag of sets $\vec{T}=T^{0} \subseteq$ $T^{1} \subseteq \cdots \subseteq T^{n}$, where $J_{T} \subseteq T^{0}$ and $T^{n} \subseteq V_{T}$.

3.8 Proposition Let $T$ be a necklace. There is a natural isomorphism of simplicial categories between $\mathfrak{C}(T)$ and $N C_{T}$.

Proof Write $T=B_{1} \vee B_{2} \vee \cdots \vee B_{k}$, where the $B_{i}$ 's are the beads of $T$. Then

$$
\mathfrak{C}(T)=\mathfrak{C}\left(B_{1}\right) \amalg_{\mathfrak{C}(*)} \mathfrak{C}\left(B_{2}\right) \amalg_{\mathfrak{C}(*)} \cdots \amalg_{\mathfrak{C}(*)} \mathfrak{C}\left(B_{k}\right)
$$


since $\mathfrak{C}$ preserves colimits. Note that $\mathfrak{C}(*)=\mathfrak{C}\left(\Delta^{0}\right)=*$, the category with one object and a single morphism (the identity).

Note that we have isomorphisms $\mathfrak{C}\left(B_{i}\right) \cong N C_{B_{i}}$ by Lemma 2.5. We therefore get maps of categories $\mathfrak{C}\left(B_{i}\right) \rightarrow N C_{B_{i}} \rightarrow N C_{T}$, and it is readily checked these extend to a map $f: \mathfrak{C}(T) \rightarrow N C_{T}$. To see that this functor is an isomorphism, it suffices to show that it is fully faithful (as it is clearly a bijection on objects).

For any $a, b \in T_{0}$ we will construct an inverse to the map $f: \mathfrak{C}(T)(a, b) \rightarrow N C_{T}(a, b)$, when $b>a$ (the case $b \leq a$ being obvious). Let $B_{r}$ and $B_{s}$ be the beads containing $a$ and $b$, respectively (if $a$ (resp. $b$ ) is a joint, let $B_{r}$ (resp. $B_{s}$ ) be the latter (resp. former) of the two beads which contain it). Let $j_{r}, j_{r+1} \ldots, j_{s+1}$ denote the ordered elements of $J_{T}(a, b)$, indexed so that $j_{i}$ and $j_{i+1}$ lie in the bead $B_{i}$; note that $j_{r}=a$ and $j_{s+1}=b$.

Any simplex $x \in N C_{T}(a, b)_{n}$ can be uniquely written as the composite of $n$-simplices $x_{s} \circ \cdots \circ x_{r}$, where $x_{i} \in N C_{T}\left(j_{i}, j_{i+1}\right)_{n}$. Now $j_{i}$ and $j_{i+1}$ are vertices within the same bead $B_{i}$ of $T$, therefore $x_{i}$ may be regarded as an $n$-simplex in $\mathfrak{C}\left(B_{i}\right)\left(j_{i}, j_{i+1}\right)$. We then get associated $n$-simplices in $\mathfrak{C}(T)\left(j_{i}, j_{i+1}\right)$, and taking their composite gives an $n$-simplex $\tilde{x} \in \mathfrak{C}(T)(a, b)$. We define a map $g: N C_{T}(a, b) \rightarrow \mathfrak{C}(T)(a, b)$ by sending $x$ to $\tilde{x}$. One readily checks that this is well-defined and compatible with the simplicial operators, and it is also clear that $f \circ g=\mathrm{id}$.

To see that $f$ is an isomorphism it suffices to now show that $g$ is surjective. From the expression (3.9) for $\mathfrak{C}(T)$ as a colimit of the categories $\mathfrak{C}\left(B_{i}\right)$, it follows at once that every map in $\mathfrak{C}(T)(a, b)$ can be written as a composite of maps from the $\mathfrak{C}\left(B_{i}\right)$ 's. It is an immediate consequence that $g$ is surjective.

3.10 Corollary Let $T=B_{0} \vee B_{1} \vee \cdots \vee B_{k}$ be a necklace. Let $a, b \in T_{0}$ be such that $a<b$. Let $j_{r}, j_{r+1}, \ldots, j_{s+1}$ be the elements of $J_{T}(a, b)$ (in order), and let $B_{i}$ denote the bead containing $j_{i}$ and $j_{i+1}$, for $r \leq i \leq s$. Then the map

$$
\mathfrak{C}\left(B_{s}\right)\left(j_{s}, j_{s+1}\right) \times \cdots \times \mathfrak{C}\left(B_{r}\right)\left(j_{r}, j_{r+1}\right) \rightarrow \mathfrak{C}(T)(a, b)
$$

is an isomorphism. Therefore $\mathfrak{C}(T)(a, b) \cong\left(\Delta^{1}\right)^{N}$ where $N=\left|V_{T}(a, b)-J_{T}(a, b)\right|$. In particular, $\mathfrak{C}(T)(a, b)$ is contractible if $a \leq b$ and empty otherwise.

Proof Follows at once from Proposition 3.8.

3.11 Remark Given a necklace $T$, there is a heuristic way to understand faces (both codimension one and higher) in the cubes $\mathfrak{C}(T)(a, b)$ in terms of "paths" from $a$ to $b$ in $T$. To choose a face in $\mathfrak{C}(T)(a, b)$, one chooses three subsets $Y, N, M \subset V_{T}(a, b)$ 
which cover the set $V_{T}(a, b)$ and are mutually disjoint. The set $Y$ is the set of vertices which we require our path to go through-it must contain $J_{T}(a, b)$; the set $N$ is the set of vertices which we require our path to not go through; and the set $M$ is the set of vertices for which we leave the question open. Such choices determine a unique face in $\mathfrak{C}(T)(a, b)$. The dimension of this face is precisely the number of vertices in $M$.

\section{The rigidification functor}

Recall that we fully understand $\mathfrak{C}\left(\Delta^{n}\right)$ as a simplicial category, and that $\mathfrak{C}: s \mathscr{S} t \rightarrow s \mathscr{C}$ at is defined for $S \in s \mathscr{S e t}$ by the formula

$$
\mathfrak{C}(S)=\operatorname{colim}_{\Delta^{n} \rightarrow S} \mathfrak{C}\left(\Delta^{n}\right) .
$$

The trouble with this formula is that given a diagram $X: I \rightarrow s^{C}$ at of simplicial categories, it is generally quite difficult to understand the mapping spaces in the colimit. For the above colimit, however, something special happens because the simplicial categories $\mathfrak{C}\left(\Delta^{n}\right)$ are "directed" in a certain sense. It turns out by making use of necklaces one can write down a precise description of the mapping spaces for $\mathfrak{C}(S)$; this is the goal of the present section.

Fix a simplicial set $S$ and elements $a, b \in S_{0}$. For any necklace $T$ and map $T \rightarrow S_{a, b}$, there is an induced map $\mathfrak{C}(T)(\alpha, \omega) \rightarrow \mathfrak{C}(S)(a, b)$. Let $\left(\mathcal{N} e c \downarrow S_{a, b}\right)$ denote the category whose objects are pairs $\left[T, T \rightarrow S_{a, b}\right]$ and whose morphisms are maps of necklaces $T \rightarrow T^{\prime}$ giving commutative triangles over $S$. Then we obtain a map

$$
\operatorname{colim}_{T \rightarrow S \in\left(\mathcal{N} e \downarrow \downarrow S_{a, b}\right)}[\mathfrak{C}(T)(\alpha, \omega)] \longrightarrow \mathfrak{C}(S)(a, b) .
$$

Let us write $E_{S}(a, b)$ for the domain of this map. Note that there are composition maps

$$
E_{S}(b, c) \times E_{S}(a, b) \longrightarrow E_{S}(a, c)
$$

induced in the following way. Given $T \rightarrow S_{a, b}$ and $U \rightarrow S_{b, c}$ where $T$ and $U$ are necklaces, one obtains $T \vee U \rightarrow S_{a, c}$ in the evident manner. The composite

$$
\begin{gathered}
\mathfrak{C}(U)\left(\alpha_{U}, \omega_{U}\right) \times \mathfrak{C}(T)\left(\alpha_{T}, \omega_{T}\right) \longrightarrow \mathfrak{C}(T \vee U)\left(\omega_{T}, \omega_{U}\right) \times \mathfrak{C}(T \vee U)\left(\alpha_{T}, \omega_{T}\right) \\
\downarrow^{\downarrow} \\
\mathfrak{C}(T \vee U)\left(\alpha_{T}, \omega_{U}\right)
\end{gathered}
$$

induces the pairing of (4.2), where the first map in the composite can be understood using Proposition 3.8 and we have used $\omega_{T}=\alpha_{U}$. One readily checks that $E_{S}$ is a 
simplicial category with object set $S_{0}$, and (4.1) yields a map of simplicial categories $E_{S} \rightarrow \mathfrak{C}(S)$. Moreover, the construction $E_{S}$ is clearly functorial in $S$.

Here is our first result:

4.3 Proposition For every simplicial set $S$, the map $E_{S} \rightarrow \mathfrak{C}(S)$ is an isomorphism of simplicial categories.

Proof First note that if $S$ is itself a necklace then the identity map $S \rightarrow S$ is a terminal object in $\left(\mathcal{N} e c \downarrow S_{a, b}\right)$. It follows at once that $E_{S}(a, b) \rightarrow \mathfrak{C}(S)(a, b)$ is an isomorphism for all $a$ and $b$.

Now let $S$ be an arbitrary simplicial set, and choose vertices $a, b \in S_{0}$. We will show that $E_{S}(a, b) \rightarrow \mathfrak{C}(S)(a, b)$ is an isomorphism. Consider the commutative diagram of simplicial sets

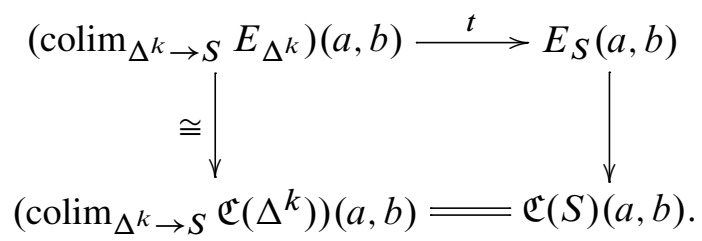

The bottom equality is the definition of $\mathfrak{C}$. The left-hand map is an isomorphism by our remarks in the first paragraph. It follows that the top map $t$ is injective. To complete the proof it therefore suffices to show that $t$ is surjective.

Choose an $n$-simplex $x \in E_{S}(a, b)_{n}$; it is represented by a necklace $T$, a map $f: T \rightarrow S_{a, b}$, and an element $\tilde{x} \in \mathfrak{C}(T)(\alpha, \omega)$. We have a commutative diagram

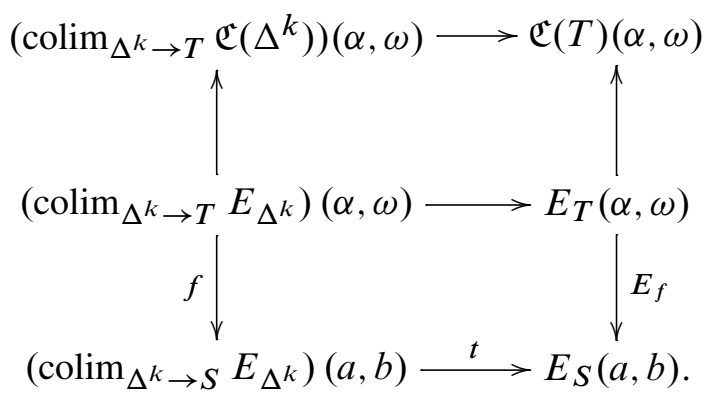

The $n$-simplex in $E_{T}(\alpha, \omega)$ represented by $\left[T, \operatorname{id}_{T}: T \rightarrow T ; \tilde{x}\right]$ is sent to $x$ under $E_{f}$. It suffices to show that the middle horizontal map is surjective, for then $x$ will be in the image of $t$. But the top map is an isomorphism, and the vertical arrows in the top row are isomorphisms by the remarks from the first paragraph. Thus, we are done. 
4.4 Corollary For any simplicial set $S$ and elements $a, b \in S_{0}$, the simplicial set $\mathfrak{C}(S)(a, b)$ admits the following description. An $n$-simplex in $\mathfrak{C}(S)(a, b)$ consists of an equivalence class of triples $[T, T \rightarrow S, \vec{T}]$, where

- $T$ is a necklace;

- $T \rightarrow S$ is a map of simplicial sets which sends $\alpha_{T}$ to $a$ and $\omega_{T}$ to $b$;

- $\vec{T}$ is a flag of sets $T^{0} \subseteq T^{1} \subseteq \cdots \subseteq T^{n}$ such that $T^{0}$ contains the joints of $T$ and $T^{n}$ is contained in the set of vertices of $T$.

The equivalence relation is generated by considering $[T, T \rightarrow S ; \vec{T}]$ and $[U, U \rightarrow S ; \vec{U}]$ to be equivalent if there exists a map of necklaces $f: T \rightarrow U$ over $S$ with $\vec{U}=f_{*}(\vec{T})$. The $i$-th face (resp. degeneracy) map omits (resp. repeats) the set $T^{i}$ in the flag. That is, if $x=\left[T, T \rightarrow S ; T^{0} \subseteq \cdots \subseteq T^{n}\right]$ represents an $n$-simplex of $\mathfrak{C}(S)(a, b)$ and $0 \leq i \leq n$, then

and

$$
s_{i}(x)=\left[T, T \rightarrow S ; T^{0} \subseteq \cdots \subseteq T^{i} \subseteq T^{i} \subseteq \cdots \subseteq T^{n}\right]
$$

$$
d_{i}(x)=\left[T \rightarrow S ; T^{0} \subseteq \cdots \subseteq T^{i-1} \subseteq T^{i+1} \subseteq \cdots \subseteq T^{n}\right] .
$$

Proof This is a straightforward interpretation of the colimit appearing in the definition of $E_{S}$ from (4.1). Recall that every colimit can be written as a coequalizer

$$
\operatorname{colim}_{T \rightarrow S \in\left(\mathcal{N e c} \downarrow S_{a, b}\right)}[\mathfrak{C}(T)(\alpha, \omega)] \cong \operatorname{coeq}\left[\coprod_{T_{1} \rightarrow T_{2} \rightarrow S} \mathfrak{C}\left(T_{1}\right)(\alpha, \omega) \rightrightarrows \coprod_{T \rightarrow S} \mathfrak{C}(T)(\alpha, \omega)\right],
$$

and that simplices of $\mathfrak{C}(T)(\alpha, \omega)$ are identified with flags of subsets of $V_{T}$, containing $J_{T}$, by Proposition 3.8. The simplices of $\bigsqcup_{T \rightarrow S} \mathfrak{C}(T)(\alpha, \omega)$ therefore correspond to triples $[T, T \rightarrow S, \vec{T}]$, and the simplices of the coequalizer correspond to equivalence classes of such triples. The relation given in the statement of the corollary is precisely the one coming from the above coequalizer.

Our next goal is to simplify the equivalence relation appearing in Corollary 4.4 somewhat. This analysis is somewhat cumbersome, but culminates in the important Proposition 4.10.

Let us begin by introducing some terminology. A flagged necklace is a pair $[T, \vec{T}]$ where $T$ is a necklace and $\vec{T}$ is a flag of subsets of $V_{T}$ which all contain $J_{T}$. The length of the flag is the number of subset symbols, or one less than the number of subsets. A morphism of flagged necklaces $[T, \vec{T}] \rightarrow[U, \vec{U}]$ exists only if the flags have the same length, in which case it is a map of necklaces $f: T \rightarrow U$ such that $f\left(T^{i}\right)=U^{i}$ for all $i$. Finally, a flag $\vec{T}=\left(T^{0} \subseteq \cdots \subseteq T^{n}\right)$ is called flanked if 
$T^{0}=J_{T}$ and $T^{n}=V_{T}$. Note that if $[T, \vec{T}]$ and $[U, \vec{U}]$ are both flanked, then every morphism $[T, \vec{T}] \rightarrow[U, \vec{U}]$ is surjective (because its image will be a subnecklace of $U$ having the same joints and vertices as $U$, hence it must be all of $U$ ).

4.5 Lemma Under the equivalence relation of Corollary 4.4, each of the triples $[T, T \rightarrow S, \vec{T}]$ is equivalent to one in which the flag is flanked. Moreover, two flanked triples are equivalent (in the sense of Corollary 4.4) if and only if they can be connected by a zigzag of morphisms of flagged necklaces in which every triple of the zigzag is flanked.

Proof Suppose given a flagged necklace $\left[T, T^{0} \subseteq \cdots \subseteq T^{n}\right]$. There is a unique subnecklace $T^{\prime} \hookrightarrow T$ whose set of joints is $T^{0}$ and whose vertex set is $T^{n}$. Then the pair $\left(T^{\prime}, T^{0} \subseteq \cdots \subseteq T^{n}\right)$ is flanked. This assignment, which we call flankification, is actually functorial: a morphism of flagged necklaces $f:[T, \vec{T}] \rightarrow[U, \vec{U}]$ must map $T^{\prime}$ into $U^{\prime}$ and therefore gives a morphism $\left[T^{\prime}, \vec{T}\right] \rightarrow\left[U^{\prime}, \vec{U}\right]$.

Using the equivalence relation of Corollary 4.4, each triple $[T, T \rightarrow S, \vec{T}]$ will be equivalent to the flanked triple $\left[T^{\prime}, T^{\prime} \rightarrow T \rightarrow S, \vec{T}\right]$ via the map $T^{\prime} \rightarrow T$. If the flanked triple $[U, U \rightarrow S, \vec{U}]$ is equivalent to the flanked triple $[V, V \rightarrow S, \vec{V}]$ then there is a zigzag of maps between triples which starts at the first and ends at the second, by Corollary 4.4. Applying the flankification functor gives a corresponding zigzag in which every object is flanked.

4.6 Remark By the previous lemma, we can alter our model for $\mathfrak{C}(S)(a, b)$ so that the $n$-simplices are equivalence classes of triples $[T, T \rightarrow S, \vec{T}]$ in which the flag is flanked, and the equivalence relation is given by maps (which are necessarily surjections) of flanked triples. Under this model the degeneracies and inner faces are given by the same description as before: repeating or omitting one of the subsets in the flag. The outer faces $d_{0}$ and $d_{n}$ are now more complicated, however, because omitting the first or last subset in the flag may produce one which is no longer flanked; one must first remove the subset and then apply the flankification functor from Lemma 4.5. This model for $\mathfrak{C}(S)(a, b)$ was originally shown to us by Jacob Lurie; it will play only a very minor role in what follows.

Our next task will be to analyze surjections of flagged triples. Let $T$ be a necklace and $S$ a simplicial set. Say that a map $T \rightarrow S$ is totally nondegenerate if the image of each bead of $T$ is a nondegenerate simplex of $S$. Note a totally nondegenerate map need not be an injection: for example, let $S=\Delta^{1} / \partial \Delta^{1}$ and consider the nondegenerate $1-$ simplex $\Delta^{1} \rightarrow S$. 
As a prelude to what we are about to do, recall that the $n$-simplices of a simplicial set $S$ correspond to maps $\Delta^{n} \rightarrow S$ in $s$ Set. Every map $\sigma: \Delta^{k} \rightarrow \Delta^{n}$ represents a simplicial operator, in the sense that if $s: \Delta^{n} \rightarrow S$ is an $n$-simplex of $S$ then $\sigma \circ s$ gives a $k$-simplex of $S$. Said differently, there is an evident map of categories $\Delta \rightarrow s \mathscr{S}$ et (the Yoneda embedding) and the image is the full subcategory of $s \mathscr{C}$ et whose objects are the $\Delta^{n}$ 's: so every map $\Delta^{k} \rightarrow \Delta^{n}$ corresponds to a map in $\Delta$, ie, a simplicial operator. Under this correspondence, surjections $\Delta^{k} \rightarrow \Delta^{n}$ correspond to degeneracy operators and injections correspond to face operators.

In a simplicial set $S$, if $z \in S$ is a degenerate simplex then there is a unique nondegenerate simplex $z^{\prime}$ and a unique degeneracy operator $s_{\sigma}=s_{i_{1}} s_{i_{2}} \cdots s_{i_{k}}$ such that $z=s_{\sigma}\left(z^{\prime}\right)$; see Hirschhorn [10, Lemma 15.8.4]. In other words, if $\Delta^{n} \rightarrow S$ is degenerate then there is a nondegenerate simplex $\Delta^{k} \rightarrow S$ and a unique surjection $\Delta^{n} \rightarrow \Delta^{k}$ making the evident triangle commute. Applying this one bead at a time, one finds that for any map $T \rightarrow S$ there is a necklace $\bar{T}$, a map $\bar{T} \rightarrow S$ which is totally nondegenerate, and a surjection of necklaces $T \rightarrow \bar{T}$ making the evident triangle commute; moreover, these three things are unique up to isomorphism.

4.7 Proposition Let $S$ be a simplicial set and let $a, b \in S_{0}$.

(a) Suppose that $T$ and $U$ are necklaces, $u: U \rightarrow S$ and $t: T \rightarrow S$ are two maps, and that $t$ is totally nondegenerate. Then there is at most one surjection $f: U \rightarrow T$ such that $u=t \circ f$.

(b) Suppose that one has a diagram

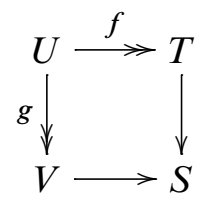

where $T, U$, and $V$ are flagged necklaces, $T \rightarrow S$ is totally nondegenerate, and $f$ and $g$ are surjections. Then there exists a unique map of flagged necklaces $V \rightarrow T$ making the diagram commute.

Proof It is easy to see that if $A \rightarrow B$ is a map of necklaces then every joint of $B$ is the image of some joint of $A$. Moreover, if $A \rightarrow B$ is surjective then one checks using the ordering of $A$ and $B$ that every joint of $A$ must map to a joint of $B$. It follows readily that if $A \rightarrow B$ is a surjection of necklaces and $B \neq *$ then every bead of $B$ is surjected on by a unique bead of $A$. Also, each bead of $A$ is either collapsed onto a joint of $B$ or else mapped surjectively onto a bead of $B$. 
For (a), note that we may assume $T \neq *$ (or else the claim is trivial). Assume there are two distinct surjections $f, f^{\prime}: U \rightarrow T$ such that $t f=t f^{\prime}=u$. Let $B$ be the first bead of $U$ on which $f$ and $f^{\prime}$ disagree. Let $j$ denote the initial vertex of $B$, and let $C$ be the bead of $T$ whose initial vertex is $f(j)=f^{\prime}(j)$.

Suppose that $f$ collapses $B$ to a point, in which case $u$ must also map $B$ to a point. Then $f^{\prime}$ cannot surject $B$ onto $C$, for otherwise $C \rightarrow S$ would factor through the point $u(B)$ and this would contradict $T \rightarrow S$ being totally nondegenerate. So $f^{\prime}$ also collapses $B$ to point, contradicting the assumption that $f$ and $f^{\prime}$ disagree on $B$. Therefore $f$ (and by symmetry $f^{\prime}$ ) cannot collapse $B$ to a point, and hence must surject $B$ onto $C$. This identifies the simplex $B \rightarrow U \rightarrow S$ with a degeneracy of the nondegenerate simplex $C \rightarrow S$. Then by uniqueness of degeneracies we have that $f$ and $f^{\prime}$ must coincide on $B$, which is a contradiction.

Next we turn to part (b). Note that the map $V \rightarrow T$ will necessarily be surjective, so the uniqueness part is guaranteed by (a); we need only show existence.

Observe that if $B$ is a bead in $U$ which maps to a point in $V$ then it maps to a point in $T$, by the reasoning above. It now follows that there exists a necklace $U^{\prime}$, obtained by collapsing every bead of $U$ that maps to a point in $V$, and a commutative diagram:

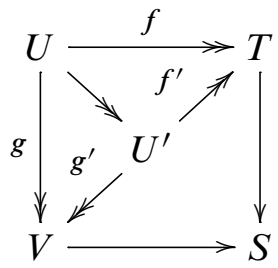

Replacing $U, f$, and $g$ by $U^{\prime}, f^{\prime}$, and $g^{\prime}$, and dropping the primes, we can now assume that $g$ induces a one-to-one correspondence between beads of $U$ and beads of $V$. Let $B_{1}, \ldots, B_{m}$ denote the beads of $U$, and let $C_{1}, \ldots, C_{m}$ denote the beads of $V$.

Assume that we have constructed the lift $l: V \rightarrow T$ on the beads $C_{1}, \ldots, C_{i-1}$. If the bead $B_{i}$ is mapped by $f$ to a point, then evidently we can define $l$ to map $C_{i}$ to this same point and the diagram will commute. Otherwise $f$ maps $B_{i}$ surjectively onto a certain bead $D$ inside of $T$. We have the diagram

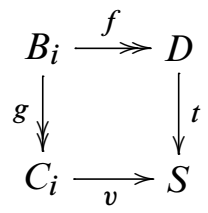

where here $f$ and $g$ are surjections between simplices and therefore represent degeneracy operators $s_{f}$ and $s_{g}$. We have that $s_{f}(t)=s_{g}(v)$. But the simplex $t$ of $S$ is 
nondegenerate by assumption, therefore by [10, Lemma 15.8.4] we must have $v=s_{h}(t)$ for some degeneracy operator $s_{h}$ such that $s_{f}=s_{g} s_{h}$. The operator $s_{h}$ corresponds (as explained prior to Proposition 4.7) to a surjection of simplices $C_{i} \rightarrow D$ making the above square commute, and we define $l$ on $C_{i}$ to coincide with this map. Continuing by induction, this produces the desired lift $l$. It is easy to see that $l$ is a map of flagged necklaces, as $l\left(V^{i}\right)=l\left(g\left(U^{i}\right)\right)=f\left(U^{i}\right)=T^{i}$.

4.8 Corollary Let $S$ be a simplicial set and $a, b \in S_{0}$. Under the equivalence relation from Corollary 4.4, every triple $\left[T, T \rightarrow S_{a, b}, \vec{T}\right]$ is equivalent to a unique triple $\left[U, U \rightarrow S_{a, b}, \vec{U}\right]$ which is both flanked and totally nondegenerate.

Proof Let $t=\left[T, T \rightarrow S_{a, b}, \vec{T}\right]$. Then $t$ is clearly equivalent to at least one flanked, totally nondegenerate triple because we can replace $t$ with $\left[T^{\prime}, T^{\prime} \rightarrow S_{a, b}, \vec{T}\right]$ (flankification) and then with $\left[\bar{T}^{\prime}, \bar{T}^{\prime} \rightarrow S_{a, b}, \vec{T}^{\prime}\right]$ (defined above Proposition 4.7).

Now suppose that $\left[U, U \rightarrow S_{a, b}, \vec{U}\right]$ and $\left[V, V \rightarrow S_{a, b}, \vec{V}\right]$ are both flanked, totally nondegenerate, and equivalent in $\mathfrak{C}(S)(a, b)_{n}$. Then by Lemma 4.5 there is a zigzag of maps between flanked necklaces (over $S$ ) connecting $U$ to $V$ :

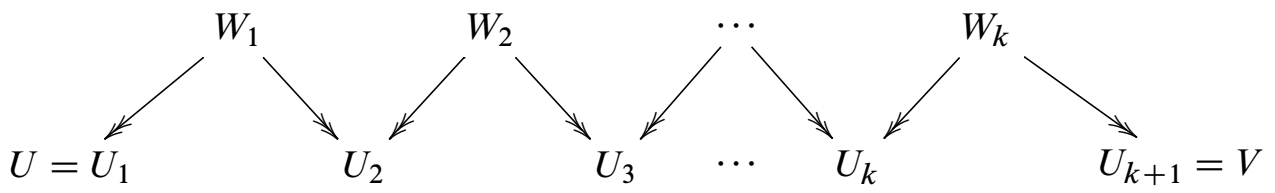

Using Proposition 4.7, we inductively construct surjections of flanked necklaces $U_{i} \rightarrow U$ over $S$. This produces a surjection $V \rightarrow U$ over $S$. Similarly, we obtain a surjection $U \rightarrow V$ over $S$. By Proposition 4.7(a) these maps must be inverses of each other; that is, they are isomorphisms.

4.9 Remark Again, as in Remark 4.6 the above corollary shows that we can describe $\mathfrak{C}(S)(a, b)$ as the simplicial set whose $n$-simplices are triples $[T, T \rightarrow S, \vec{T}]$ which are both flanked and totally nondegenerate. The degeneracies and inner faces are again easy to describe - they are repetition or omission of a set in the flag-but for the outer faces one must first omit a set and then modify the triple appropriately. The usefulness of this description is limited because of these complications with the outer faces, but it does make a brief appearance in Corollary 4.13 below.

The following result is the culmination of our work in this section, and will turn out to be a key step in the proof of our main theorems. Fix a simplicial set $S$ and vertices $a, b \in S_{0}$, and let $F_{n}$ denote the category of flagged triples over $S_{a, b}$ that have length $n$. That is, the objects of $F_{n}$ are triples $\left[T, T \rightarrow S_{a, b}, T^{0} \subseteq \cdots \subseteq T^{n}\right]$ and morphisms are maps of necklaces $f: T \rightarrow T^{\prime}$ over $S$ such that $f\left(T^{i}\right)=\left(T^{\prime}\right)^{i}$ for all $i$. 
4.10 Proposition For each $n \geq 0$, the nerve of $F_{n}$ is homotopy discrete in $s \mathscr{S e t}_{K}$.

Proof Recall from Lemma 4.5 that there is a functor $\phi: F_{n} \rightarrow F_{n}$ which sends any triple to its "flankification". The flankification is a subnecklace of the original necklace, and therefore the inclusion gives a natural transformation from $\phi$ to the identity. If $F_{n}^{\prime}$ denotes the full subcategory of $F_{n}$ consisting of flanked triples, we thus find that $F_{n}^{\prime} \hookrightarrow F_{n}$ induces a Kan equivalence upon taking nerves. To prove the proposition it will therefore suffice to prove that the nerve of $F_{n}^{\prime}$ is homotopy discrete.

Recall from Corollary 4.8 that every component of $F_{n}^{\prime}$ contains a unique triple $t$ which is both flanked and totally nondegenerate. Moreover, following the proof of that corollary one sees that every triple in the same component as $t$ admits a unique map to $t$-uniqueness follows from Proposition 4.7(a), using that a map of flanked triples is necessarily surjective. We have therefore shown that $t$ is a final object for its component, hence its component is contractible. This completes the proof.

\subsection{The functor $\mathfrak{C}$ applied to ordered simplicial sets}

Note that even if a simplicial set $S$ is small—say, in the sense that it has finitely many nondegenerate simplices - the space $\mathfrak{C}(S)(a, b)$ may be quite large. This is due to the fact that there are infinitely many necklaces mapping to $S$ (if $S$ is nonempty). For certain simplicial sets $S$, however, it is possible to restrict to necklaces which map injectively into $S$; this cuts down the possibilities. The following results and subsequent example demonstrate this. Recall the definition of ordered simplicial sets from Definition 3.2.

4.12 Lemma Let $D$ be an ordered simplicial set and let $a, b \in D_{\underline{0}}$. Then every $n$-simplex in $\mathfrak{C}(D)(a, b)$ is represented by a unique triple $[T, T \rightarrow D, \vec{T}]$ in which $T$ is a necklace, $\vec{T}$ is a flanked flag of length $n$, and the map $T \rightarrow D$ is injective.

Proof By Corollary 4.8, every $n$-simplex in $\mathfrak{C}(D)(a, b)$ is represented by a unique triple $[T, T \rightarrow D, \vec{T}]$ which is both flanked and totally nondegenerate. It suffices to show that if $D$ is ordered, then any totally nondegenerate map $T \rightarrow D$ is injective. This follows from Lemma 3.3(6).

4.13 Corollary Let $D$ be an ordered simplicial set, and $a, b \in D_{0}$. Let $M_{D}(a, b)$ denote the simplicial set for which $M_{D}(a, b)_{n}$ is the set of triples $\left[T, f: T \rightarrow D_{a, b}, \vec{T}\right]$, where $f$ is injective and $\vec{T}$ is a flanked flag of length $n$; face and boundary maps are as in Remark 4.6. Then there is a natural isomorphism

$$
\mathfrak{C}(D)(a, b) \stackrel{\cong}{\longrightarrow} M_{D}(a, b) .
$$

Proof This follows immediately from Lemma 4.12. 
4.14 Example Consider the simplicial set $S=\Delta^{2} \amalg_{\Delta^{1}} \Delta^{2}$ depicted as follows:

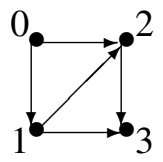

We will describe the mapping space $X=\mathfrak{C}(S)(0,3)$ by giving its nondegenerate simplices and face maps. Note that $S$ is ordered.

By Lemma 4.12, it suffices to consider flanked necklaces that inject into $S$. There are only five such injections that have endpoints 0 and 3 . These are $T=\Delta^{1} \vee \Delta^{1}$, which maps to $S$ in two different ways $f, g$; and $U=\Delta^{1} \vee \Delta^{1} \vee \Delta^{1}, V=\Delta^{1} \vee \Delta^{2}$, and $W=\Delta^{2} \vee \Delta^{1}$, each of which maps uniquely into $S_{0,3}$. The image of $T_{0}$ under $f$ is $\{0,1,3\}$ and under $g$ is $\{0,2,3\}$. The images of $U_{0}, V_{0}$, and $W_{0}$ are all $\{0,1,2,3\}$.

We find that the set $X_{0}$ consists of three elements $[T ;\{0,1,3\}],[T ;\{0,2,3\}]$ and $[U ;\{0,1,2,3\}]$. There are two nondegenerate 1 -simplices, $[V ;\{0,1,3\} \subset\{0,1,2,3\}]$ and $[W ;\{0,2,3\} \subset\{0,1,2,3\}]$. These connect the three 0 -simplices in the obvious way, resulting in two 1 -simplices with a common final vertex. There are no higher nondegenerate simplices. Thus $\mathfrak{C}(S)(0,3)$ looks like

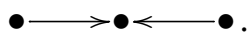

\section{Homotopical models for rigidification}

In the last section we gave a very explicit description of the mapping spaces $\mathfrak{C}(S)(a, b)$, for arbitrary simplicial sets $S$ and $a, b \in S_{0}$. While this description was explicit, in some ways it is not very useful from a homotopical standpoint-in practice it is hard to use this description to identify the homotopy type of $\mathfrak{C}(S)(a, b)$.

In this section we will discuss a functor $\mathfrak{C}^{\text {nec }}: s \mathscr{S} e t \rightarrow s \mathscr{C}$ at that has a simpler description than $\mathfrak{C}$ and which is more homotopical. We prove that for any simplicial set $S$ there is a natural zigzag of weak equivalences between $\mathfrak{C}(S)$ and $\mathfrak{C}^{\text {nec }}(S)$. Variants of this construction are also introduced, leading to a collection of functors $s \mathscr{S} e t \rightarrow s \mathrm{Cat}$ all of which are weakly equivalent to $\mathfrak{C}$.

Let $S \in s \mathscr{S}_{e t}$. A choice of $a, b \in S_{0}$ will be regarded as a map $\partial \Delta^{1} \rightarrow S$. Let $\left(\mathcal{N e c} \downarrow S_{a, b}\right)$ be the overcategory for the inclusion functor $\mathcal{N e c} \hookrightarrow\left(\partial \Delta^{1} \downarrow S\right)$. Finally, define

$$
\mathfrak{C}^{\text {nec }}(S)(a, b)=N\left(\mathcal{N e c} \downarrow S_{a, b}\right) .
$$

Then $\mathfrak{C}(S)$ is a simplicial category in an evident way; see (1.2). 
5.1 Remark Both the functor $\mathfrak{C}$ and the functor $\mathfrak{C}^{\text {nec }}$ have distinct advantages and disadvantages. The main advantage to $\mathfrak{C}$ is that it is left adjoint to the coherent nerve functor $N$ (in fact it is a left Quillen functor $s \mathscr{S e t}_{J} \rightarrow s_{\mathrm{bat}}$ ); as such, it preserves colimits. However, as mentioned above, the functor $\mathfrak{C}$ can be difficult to use in practice because the mapping spaces have an awkward description.

It is at this point that our functor $\mathfrak{C}^{\text {nec }}$ becomes useful, because the mapping spaces are given as nerves of 1 -categories. Many tools are available for determining when a morphism between nerves is a Kan equivalence. This will be an important point in [8], where we show the $\mathfrak{C}$ functor gives a Quillen equivalence between $s \mathscr{S}_{e} t_{J}$ and $s \mathscr{C a t}_{\text {at }}$ See also Section 6 below.

Our main theorem is that there is a simple zigzag of weak equivalences between $\mathfrak{C}(S)$ and $\mathfrak{C}^{\text {nec }}(S)$; that is, there is a functor $\mathfrak{C}^{\text {hoc }}: s \mathscr{S e t} \rightarrow s \mathscr{b}$ at and natural weak equivalences $\mathfrak{C} \leftarrow \mathfrak{C}^{\text {hoc }} \rightarrow \mathfrak{C}^{\text {nec }}$. We begin by describing the functor $\mathfrak{C}^{\text {hoc }}$.

Fix a simplicial set $S$. Define $\mathfrak{C}^{\text {hoc }}(S)$ to have object set $S_{0}$, and for every $a, b \in S_{0}$,

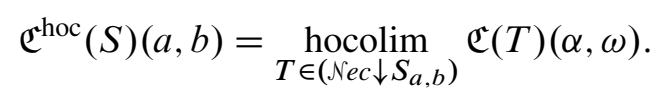

Note the similarities to Proposition 4.3, where it was shown that $\mathfrak{C}(S)(a, b)$ has a similar description in which the hocolim is replaced by the colim. In our definition of $\mathfrak{C}^{\text {hoc }}(S)(a, b)$ we mean to use a particular model for the homotopy colimit: namely, recall that if $X: I \rightarrow s \mathscr{S e t}$ then the Bousfield-Kan model [4, XII.5.2] for hocolim $I X$ is the diagonal of the bisimplicial set (the simplicial replacement of $X$ ) which in level $k$ is

$$
\coprod_{i_{0} \rightarrow \cdots \rightarrow i_{k}} X_{i_{0}} .
$$

Applying this in our case, $\mathfrak{C}^{\text {hoc }}(S)(a, b)$ is the diagonal of the bisimplicial set whose $(k, l)$-simplices are pairs

$$
\left(F:[k] \rightarrow\left(\mathcal{N} e c \downarrow S_{a, b}\right), \quad x \in \mathfrak{C}(F(0))(\alpha, \omega)_{l}\right),
$$

where $F(0)$ denotes the necklace obtained by applying $F$ to $0 \in[k]$ and then applying the forgetful functor $\left(\mathcal{N} e c \downarrow S_{a, b}\right) \rightarrow \mathcal{N} e c$. The composition law for $\mathfrak{C}^{\text {hoc }}$ is defined just as for the $E_{S}$ construction from Section 4 .

If one pictures the bisimplicial set of (5.2) with the index $k$ varying horizontally and $l$ varying vertically, note that the $l$-th horizontal row is the nerve $N F_{l}$ of the category of flagged necklaces mapping to $S$ (where the flags have length $l$ ).

We proceed to establish natural transformations $\mathfrak{C}^{\text {hoc }} \rightarrow \mathfrak{C}^{\text {nec }}$ and $\mathfrak{C}^{\text {hoc }} \rightarrow \mathfrak{C}$. Note that the simplicial set $\mathfrak{C}^{\text {nec }}(S)(a, b)$ is the homotopy colimit of the constant functor 
$\{*\}:\left(\mathcal{N} e c \downarrow S_{a, b}\right) \rightarrow s \mathscr{S} t$ which sends everything to a point (using [10, Proposition 18.1.6], for example). The map $\mathfrak{C}^{\text {hoc }}(S)(a, b) \rightarrow \mathfrak{C}^{\text {nec }}(S)(a, b)$ is the map of homotopy colimits induced by the evident map of diagrams. Since the spaces $\mathfrak{C}(T)(\alpha, \omega)$ are all contractible simplicial sets (see Corollary 3.10), the induced map $\mathfrak{C}^{\text {hoc }}(S)(a, b) \rightarrow$ $\mathfrak{C}^{\text {nec }}(S)(a, b)$ is a Kan equivalence. We thus obtain a natural weak equivalence of simplicial categories $\mathfrak{C}^{\text {hoc }}(S) \rightarrow \mathfrak{C}^{\text {nec }}(S)$.

For any diagram in a model category there is a canonical natural transformation from the homotopy colimit to the colimit of that diagram. Hence there is a morphism

$$
\mathfrak{C}^{\text {hoc }}(S)(a, b) \rightarrow \operatorname{colim}_{T \in\left(\mathcal{N} e c \downarrow S_{a, b}\right)} \mathfrak{C}(T)(\alpha, \omega) \cong \mathfrak{C}(S)(a, b) .
$$

(For the isomorphism we are using Proposition 4.3.) As this is natural in $a, b \in S_{0}$ and natural in $S$, we have a natural transformation $\mathfrak{C}^{\text {hoc }} \rightarrow \mathfrak{C}$.

5.3 Theorem For every simplicial set $S$, the maps $\mathfrak{C}(S) \leftarrow \mathfrak{C}^{\text {hoc }}(S) \rightarrow \mathfrak{C}^{\text {nec }}(S)$ are weak equivalences of simplicial categories. Equivalently, the maps induce Kan equivalences on all mapping spaces.

Proof We have already established the result for $\mathfrak{C}^{\text {hoc }} \rightarrow \mathfrak{C}^{\text {nec }}$, so it suffices to show that for each simplicial set $S$ and objects $a, b \in S_{0}$ the natural map $\mathfrak{C}^{\text {hoc }}(S)(a, b) \rightarrow$ $\mathfrak{C}(S)(a, b)$ is a Kan equivalence.

Recall that $\mathfrak{C}^{\text {hoc }}(S)(a, b)$ is the diagonal of the bisimplicial set (5.2), and that the $l$-th "horizontal" row is the nerve $N F_{l}$ of the category of flagged necklaces mapping to $S$, where the flags have length $l$. Also recall from Corollary 4.4 that $\mathfrak{C}(S)(a, b)$ is the simplicial set which in level $l$ is $\pi_{0}\left(N F_{l}\right)$. But Proposition 4.10 says that $N F_{l} \rightarrow$ $\pi_{0}\left(N F_{l}\right)$ is a Kan equivalence, for every $l$. It follows that $\mathfrak{C}^{\text {hoc }}(S)(a, b) \rightarrow \mathfrak{C}(S)(a, b)$ is also a Kan equivalence.

\subsection{Other models for rigidification}

One can imagine variations of our basic construction in which one replaces necklaces with other convenient simplicial sets_-which we might term "gadgets," for lack of a better word. We will see in Section 6, for instance, that using products of necklaces leads to a nice theorem about the rigidification of a product. In [8] several key arguments will hinge on a clever choice of what gadgets to use. In the material below we give some basic requirements of the "gadgets" which will ensure they give a model equivalent to that of necklaces.

Suppose $\mathscr{P}$ is a subcategory of $s \mathscr{S} e t_{*, *}=\left(\partial \Delta^{1} \downarrow s \mathscr{S e t}\right)$ containing the terminal object. For any simplicial set $S$ and vertices $a, b \in S_{0}$, let $\left(\mathscr{P} \downarrow S_{a, b}\right)$ denote the overcategory 
whose objects are pairs $[P, P \rightarrow S]$, where $P \in \mathscr{P}$ and the map $P \rightarrow S$ sends $\alpha_{P} \mapsto a$ and $\omega_{P} \mapsto b$. Define

$$
\mathfrak{C}^{\mathscr{P}}(S)(a, b)=N\left(\mathscr{P} \downarrow S_{a, b}\right) .
$$

The object $\mathfrak{C}^{\mathscr{P}}$ is simply an assignment which takes a simplicial set $S$ with two distinguished vertices and produces a " $\mathscr{P}$-mapping space." However, if $\mathscr{P}$ is closed under the wedge operation (ie for any $P_{1}, P_{2} \in \mathscr{P}$ one has $P_{1} \vee P_{2} \in \mathscr{P}$ ), then $\mathfrak{C}^{\mathscr{P}}$ may be given the structure of a functor $s \mathscr{S}$ et $\rightarrow s \mathscr{C}$ at in the evident way.

5.5 Definition We call a subcategory $\mathcal{G} \subseteq s \mathscr{S e t}_{*, *}$ a category of gadgets if it satisfies the following properties:

(1) $\mathcal{G}$ contains the category $\mathcal{N e c}$.

(2) For every object $X \in \mathcal{G}$ and every necklace $T$, all maps $T \rightarrow X$ are contained in $\mathcal{G}$.

(3) For any $X \in \mathcal{G}$, the simplicial set $\mathfrak{C}(X)(\alpha, \omega)$ is contractible.

The category $\mathcal{G}$ is said to be closed under wedges if the following also true:

(4) For any $X, Y \in \mathcal{G}$, the wedge $X \vee Y$ also belongs to $\mathcal{G}$.

5.6 Proposition Let $\mathcal{G}$ be a category of gadgets. Then for any simplicial set $S$ and any $a, b \in S_{0}$, the natural map

$$
\mathfrak{C}^{\text {nec }}(S)(a, b) \longrightarrow \mathfrak{C}^{\mathcal{G}}(S)(a, b)
$$

(induced by the inclusion $\mathcal{N e c} \hookrightarrow \mathcal{G}$ ) is a Kan equivalence. If $\mathcal{G}$ is closed under wedges then the map of simplicial categories $\mathfrak{C}^{\text {nec }}(S) \rightarrow \mathfrak{C}^{\mathcal{G}}(S)$ is a weak equivalence.

Proof Let $j:\left(\mathcal{N e c} \downarrow S_{a, b}\right) \rightarrow\left(\mathcal{G} \downarrow S_{a, b}\right)$ be the functor induced by the inclusion map $\mathcal{N e c} \hookrightarrow \mathcal{G}$. The map in the statement of the proposition is just the nerve of $j$. To verify that it is a Kan equivalence, it is enough by Quillen's Theorem A [15] to verify that all the overcategories of $j$ are contractible. So fix an object $[X, X \rightarrow S]$ in $\left(\mathcal{G} \downarrow S_{a, b}\right)$. The overcategory $(j \downarrow[X, X \rightarrow S])$ is precisely the category $\left(\mathcal{N} e c \downarrow X_{\alpha, \omega}\right)$, the nerve of which is $\mathfrak{C}^{\text {nec }}(X)(\alpha, \omega)$. By Theorem 5.3 this nerve is weakly equivalent to $\mathfrak{C}(X)(\alpha, \omega)$, which is contractible by our assumptions about $\mathcal{G}$.

The second statement of the result is a direct consequence of the first.

\section{Properties of rigidification}

In this section we establish two main properties of the rigidification functor $\mathfrak{C}$. First, we prove that there is a natural weak equivalence $\mathfrak{C}(X \times Y) \simeq \mathfrak{C}(X) \times \mathfrak{C}(Y)$. Second, we prove that whenever $S \rightarrow S^{\prime}$ is a Joyal equivalence it follows that $\mathfrak{C}(S) \rightarrow \mathfrak{C}\left(S^{\prime}\right)$ 
is a weak equivalence in $s^{6}$ at. These properties are also proven in [13], but the proofs we give here are of a different nature and make central use of the $\mathfrak{C}^{\text {nec }}$ functor.

If $T_{1}, \ldots, T_{n}$ are necklaces then they are, in particular, ordered simplicial sets in the sense of Definition 3.2. So $T_{1} \times \cdots \times T_{n}$ is also ordered, by Lemma 3.3. Let $\mathcal{G}$ be the full subcategory of $s \mathscr{S} t_{*, *}=\left(\partial \Delta^{1} \downarrow s \mathscr{S e t}\right)$ whose objects are products of necklaces with a map $f: \partial \Delta^{1} \rightarrow T_{1} \times \cdots \times T_{n}$ that has $f(0) \preceq f(1)$.

6.1 Proposition The category $\mathcal{G}$ is a category of gadgets in the sense of Definition 5.5.

For the proof of this one needs to verify that $\mathfrak{C}\left(T_{1} \times \cdots \times T_{n}\right)(\alpha, \omega) \simeq *$. This is not difficult, but is a bit of a distraction; we prove it later as Proposition A.6.

6.2 Proposition For any simplicial sets $X$ and $Y$, both $\mathfrak{C}(X \times Y)$ and $\mathfrak{C}(X) \times \mathfrak{C}(Y)$ are simplicial categories with object set $X_{0} \times Y_{0}$. For any $a_{0}, b_{0} \in X$ and $a_{1}, b_{1} \in Y$, the natural map

$$
\mathfrak{C}(X \times Y)\left(a_{0} a_{1}, b_{0} b_{1}\right) \rightarrow \mathfrak{C}(X)\left(a_{0}, b_{0}\right) \times \mathfrak{C}(Y)\left(a_{1}, b_{1}\right)
$$

induced by $\mathfrak{C}(X \times Y) \rightarrow \mathfrak{C}(X)$ and $\mathfrak{C}(X \times Y) \rightarrow \mathfrak{C}(Y)$ is a Kan equivalence. Consequently, the map of simplicial categories

$$
\mathfrak{C}(X \times Y) \rightarrow \mathfrak{C}(X) \times \mathfrak{C}(Y)
$$

is a weak equivalence in $s$ bat.

Proof Let $\mathcal{G}$ denote the above category of gadgets, in which the objects are products of necklaces. By Theorem 5.3 and Proposition 5.6 it suffices to prove the result for $\mathfrak{C}^{\mathcal{G}}$ in place of $\mathfrak{C}$.

Consider the functors

$$
\left(\mathcal{G} \downarrow(X \times Y)_{a_{0} a_{1}, b_{0} b_{1}}\right) \underset{\theta}{\stackrel{\phi}{\rightleftarrows}}\left(\mathcal{G} \downarrow X_{a_{0}, b_{0}}\right) \times\left(\mathcal{G} \downarrow Y_{a_{1}, b_{1}}\right)
$$

given by $\quad \phi:[G, G \rightarrow X \times Y] \mapsto([G, G \rightarrow X \times Y \rightarrow X],[G, G \rightarrow X \times Y \rightarrow Y])$

$$
\theta:([G, G \rightarrow X],[H, H \rightarrow Y]) \mapsto[G \times H, G \times H \rightarrow X \times Y] .
$$

Note that we are using that the subcategory $\mathcal{G}$ is closed under finite products.

It is very easy to see that there is a natural transformation id $\rightarrow \theta \phi$, obtained by using diagonal maps, and a natural transformation $\phi \theta \rightarrow$ id, obtained by using projections. As a consequence, the maps $\theta$ and $\phi$ induce inverse homotopy equivalences on the nerves. This completes the proof. 
Recall from Section 2 that if $S$ is a set then $\mathscr{E}(S)$ is the groupoid with object set $S$ having a unique map between any two objects. Let $E: \mathscr{Y e t} \rightarrow s \mathscr{S e t}$ be given by $E(S)=$ $N(\mathscr{E}(S))$. The $k$-simplices of $E(S)$ may be identified with $(k+1)$-tuples of elements of $S$, and the face and degeneracy operators are given by deletion or repetition of entries. One may check that for any simplicial set $X$ we have $s \mathscr{S e t}(X, E(S))=\operatorname{Set}\left(X_{0}, S\right)$. In particular, taking $X=\Delta^{1}$ note that any two 0 -simplices in $E(S)$ have a unique 1 -simplex from the first to the second.

The functor $E$ is commonly called the 0 -coskeleton functor (see [1]). For any $n \in \mathbb{N}$ we denote $E^{n}=E(\{0,1, \ldots, n\})$.

Recall also from Section 2 the notion of weak equivalence for simplicial categories. In particular, note that if $\mathscr{b}$ is simplicial category then the map $\mathscr{b} \rightarrow *$ is a weak equivalence if and only if for all $a, b \in \mathrm{ob} \mathscr{C}$ the mapping space $\mathscr{C}(a, b)$ is contractible.

6.3 Lemma For any $n \geq 0$, the simplicial category $\mathfrak{C}\left(E^{n}\right)$ is contractible in $s$ bat .

Proof By Theorem 5.3 it is sufficient to prove that the mapping space $\mathfrak{C}^{\text {nec }}\left(E^{n}\right)(i, j)$ is contractible, for every $i, j \in\{0,1, \ldots, n\}$. This mapping space is the nerve of the overcategory ( $\left.\mathcal{N e c} \downarrow E_{i, j}^{n}\right)$.

Observe that if $T$ is a necklace then any map $T \rightarrow E^{n}$ extends uniquely over $\Delta[T]$. This is because maps into $E^{n}$ are determined by what they do on the 0 -skeleton, and $T \hookrightarrow \Delta[T]$ is an isomorphism on 0 -skeleta.

Consider two functors

$$
\begin{aligned}
& f, g:\left(\mathcal{N e c} \downarrow E_{i, j}^{n}\right) \rightarrow\left(\mathcal{N e c} \downarrow E_{i, j}^{n}\right) \\
& f:\left[T, T \stackrel{x}{\longrightarrow} E^{n}\right] \mapsto\left[\Delta[T], \Delta[T] \stackrel{\bar{x}}{\longrightarrow} E^{n}\right] \\
& g:\left[T, T \stackrel{x}{\longrightarrow} E^{n}\right] \mapsto\left[\Delta^{1}, \Delta^{1} \stackrel{z}{\longrightarrow} E^{n}\right] .
\end{aligned}
$$

Here $\bar{x}$ is the unique extension of $x$ to $\Delta[T]$, and $z$ is the unique $1-$ simplex of $E^{n}$ connecting $i$ to $j$. Observe that $g$ is a constant functor.

It is easy to see that there are natural transformations id $\rightarrow f \leftarrow g$. The functor $g$ factors through the terminal category $\{*\}$, so after taking nerves the identity map is null homotopic. Hence $\left(\mathcal{N e c} \downarrow E_{i, j}^{n}\right)$ is contractible.

For completeness (and because it is short) we include the following lemma, established in [13, Proof of 2.2.5.1]: 


\subsection{Lemma The functor $\mathfrak{C}: s \mathscr{S} t \rightarrow s^{C}$ at takes monomorphism to cofibrations.}

Proof Every monomorphism in $s \mathscr{Y}$ et is obtained by compositions and cobase changes from boundary inclusions of simplices. It therefore suffices to show that for each $n \geq 0$ the map $\mathfrak{C}\left(\partial \Delta^{n}\right) \rightarrow \mathfrak{C}\left(\Delta^{n}\right)$ is a cofibration in $s^{C}$ at. Let $0 \leq i, j \leq n$. If $i>0$ or $j<n$ then every map $T \rightarrow \Delta_{i, j}^{n}$, where $T$ is a necklace, actually factors through $\partial \Delta_{i, j}^{n}$. It follows that $\left(\mathcal{N e c} \downarrow \Delta_{i, j}^{n}\right) \cong\left(\mathcal{N} e c \downarrow \partial \Delta_{i, j}^{n}\right)$, and therefore

$$
\mathfrak{C}\left(\partial \Delta^{n}\right)(i, j) \rightarrow \mathfrak{C}\left(\Delta^{n}\right)(i, j)
$$

is an isomorphism by Proposition 4.3. When $i=0$ and $j=n$, the simplicial set $\mathfrak{C}\left(\Delta^{n}\right)(i, j)$ is identified with the cube $\left(\Delta^{1}\right)^{n-1}$ by Lemma 2.5 , and it is easy to see that $\mathfrak{C}\left(\partial \Delta^{n}\right)(i, j)$ is precisely the boundary of this cube.

To summarize the above paragraph, the mapping spaces in $\mathfrak{C}\left(\partial \Delta^{n}\right)$ and $\mathfrak{C}\left(\Delta^{n}\right)$ are identical except for the mapping space from 0 to $n$, and in that case the inclusion of mapping spaces is the boundary inclusion for the cube $\left(\Delta^{1}\right)^{n-1}$. Let $b$ denote this boundary inclusion.

Let $U: s \mathscr{Y}$ et $\rightarrow s$ Cat denote the functor which sends a simplicial set $S$ to the unique simplicial category $U(S)$ with two objects $x, y$ and morphisms $\operatorname{Hom}(x, x)=\operatorname{Hom}(y, y)=$ $\{*\}, \operatorname{Hom}(y, x)=\varnothing$, and $\operatorname{Hom}(x, y)=S$. There is an evident map $U\left(\partial\left(\left(\Delta^{1}\right)^{n-1}\right)\right) \rightarrow$ $\mathfrak{C}\left(\partial \Delta^{n}\right)$ sending $x$ to 0 and $y$ to $n$, and pushing out $U(b)$ along this map precisely gives $\mathfrak{C}\left(\Delta^{n}\right)$.

In view of the generating cofibrations for $s^{c}$ at (see [3]), it is easy to show that $U$ takes monomorphisms to cofibrations. Hence $U(b)$ is a cofibration. Since our map $\mathfrak{C}\left(\partial \Delta^{n}\right) \rightarrow \mathfrak{C}\left(\Delta^{n}\right)$ is a pushout of $U(b)$, it too is a cofibration.

Recall that a simplicial category is fibrant in $s$ bat if all its mapping spaces are Kan fibrant.

6.5 Lemma If $\mathscr{D}$ is a fibrant simplicial category then $N \mathscr{D}$ is a quasi-category.

Proof By adjointness it suffices to show that each $\mathfrak{C}\left(j^{n, k}\right)$ is an acyclic cofibration in $s$ bat, where $j^{n, k}: \Lambda_{k}^{n} \hookrightarrow \Delta^{n}$ is an inner horn inclusion $(0<k<n)$. It is a cofibration by Lemma 6.4 , so we must only verify that it is a weak equivalence. Just as in the proof of 6.4 above, $\mathfrak{C}\left(\Lambda_{k}^{n}\right)(i, j) \rightarrow \mathfrak{C}\left(\Delta^{n}\right)(i, j)$ is an isomorphism unless $i=0$ and $j=n$. It only remains to show that $\mathfrak{C}\left(\Lambda_{k}^{n}\right)(0, n) \rightarrow \mathfrak{C}\left(\Delta^{n}\right)(0, n)$ is a Kan equivalence.. An analysis as in Example 4.14 identifies $\mathfrak{C}\left(\Lambda_{k}^{n}\right)(0, n)$ with the result of removing one face from the boundary of $\left(\Delta^{1}\right)^{n-1}$, which clearly has the same homotopy type as the cube $\left(\Delta^{1}\right)^{n-1}$. 
6.6 Proposition If $S \rightarrow S^{\prime}$ is a map of simplicial sets which is a Joyal equivalence then $\mathfrak{C}(S) \rightarrow \mathfrak{C}\left(S^{\prime}\right)$ is a weak equivalence of simplicial categories.

Proof For any simplicial set $X$, the map $\mathfrak{C}\left(X \times E^{n}\right) \rightarrow \mathfrak{C}(X)$ induced by projection is a weak equivalence in $s \mathfrak{b}$ at. This follows by combining Proposition 6.2 with Lemma 6.3:

$$
\mathfrak{C}\left(X \times E^{n}\right) \stackrel{\sim}{\longrightarrow} \mathfrak{C}(X) \times \mathfrak{C}\left(E^{n}\right) \stackrel{\sim}{\longrightarrow} \mathfrak{C}(X) .
$$

Since $X \amalg X \hookrightarrow X \times E^{1}$ is a cofibration in s乌et, $\mathfrak{C}(X) \amalg \mathfrak{C}(X)=\mathfrak{C}(X \amalg X) \rightarrow$ $\mathfrak{C}\left(X \times E^{1}\right)$ is a cofibration in $s \mathfrak{C} a t$, by Lemma 6.4. It follows that $\mathfrak{C}\left(X \times E^{1}\right)$ is a cylinder object for $\mathfrak{C}(X)$ in $s_{\mathfrak{C} a t}$. So if $\mathscr{D}$ is a fibrant simplicial category we may compute homotopy classes of maps $[\mathfrak{C}(X), \mathscr{D}]$ as the coequalizer

$$
\operatorname{coeq}\left(s \mathscr{C a t}\left(\mathfrak{C}\left(X \times E^{1}\right), \mathscr{D}\right) \rightrightarrows s \mathscr{b a t}(\mathfrak{C}(X), \mathscr{D})\right) \text {. }
$$

But using the adjunction, this is isomorphic to

$$
\operatorname{coeq}\left(s \mathscr{S e t}\left(X \times E^{1}, N \mathscr{D}\right) \rightrightarrows \operatorname{s} \mathscr{S e t}(X, N \mathscr{D})\right) \text {. }
$$

The above coequalizer is $[X, N \mathscr{D}]_{E^{1}}$, and we have identified

$$
[\mathfrak{C}(X), \mathscr{D}] \cong[X, N \mathscr{D}]_{E^{1}} .
$$

Now let $S \rightarrow S^{\prime}$ be a Joyal equivalence. Then $\mathfrak{C}(S) \rightarrow \mathfrak{C}\left(S^{\prime}\right)$ is a map between cofibrant objects of $s^{C}$ at. To prove that it is a weak equivalence in $s^{\mathscr{C}}$ at it is sufficient to prove that the induced map on homotopy classes

$$
\left[\mathfrak{C}\left(S^{\prime}\right), \mathscr{D}\right] \rightarrow[\mathfrak{C}(S), \mathscr{D}]
$$

is a bijection, for every fibrant object $\mathscr{D} \in S \mathscr{C}$ at. Since $N \mathscr{D}$ is a quasi-category by Lemma 6.5 and $S \rightarrow S^{\prime}$ is a Joyal equivalence, we have that $\left[S^{\prime}, N \mathscr{D}\right]_{E^{1}} \rightarrow[S, N \mathscr{D}]_{E^{1}}$ is a bijection; the result then follows by (6.7).

6.8 Remark In fact it turns out that a map of simplicial sets $S \rightarrow S^{\prime}$ is a Joyal equivalence if and only if $\mathfrak{C}(S) \rightarrow \mathfrak{C}\left(S^{\prime}\right)$ is a weak equivalence of simplicial categories. This was proven in [13], and will be reproven in [8] using an extension of the methods from the present paper.

\section{Appendix A Leftover proofs}

In this section we give two proofs which were postponed in the body of the paper. 


\section{A.1 Products of necklaces}

Our first goal is to prove Proposition 6.1. Let $T_{1}, \ldots, T_{n}$ be necklaces, and consider the product $X=T_{1} \times \cdots \times T_{n}$. The main thing we need to prove is that whenever $a \preceq_{X} b$ in $X$ the mapping space $\mathfrak{C}(X)(a, b) \simeq *$ is contractible. For this we use two lemmas.

Let $Z$ be an ordered simplicial set, and let $u, v \in Z$. Suppose there is a finite set of 0-simplices $A=\left\{a_{1}, \ldots, a_{n}\right\}$ of $Z$, and we know that every inclusion $T \hookrightarrow Z_{u, v}$, where $T$ is a necklace, has at least one joint lying in $A$. It is useful to think of $T \hookrightarrow Z_{u, v}$ as a "generalized path" from $u$ to $v$, and of the vertices in $A$ as "gates". Our assumption is that every generalized path must pass through at least one gate. One can then stratify all such paths, according to which gates they pass through. We will explain a way to understand the homotopy type of $\mathfrak{C}(Z)(u, v)$ by writing it as a homotopy colimit of "smaller" spaces associated to this stratification.

To this end, consider the poset $A_{0}$ of vertices of $A$ under the relation $\preceq$ (this is a poset because $A$ is an ordered simplicial set). Let $P$ denote the collection of linearly ordered subsets $S$ of $A_{0}$ having the property that $u \preceq s \preceq v$ for all $s \in S$. That is, each element of $P$ is a chain $u \preceq s_{1} \preceq \cdots \preceq s_{n} \preceq v$ where each $s_{i} \in A$. We regard $P$ as a category, where the maps are inclusions. Also let $P_{0}$ denote the subcategory of $P$ consisting of all subsets except $\varnothing$.

Define a functor $H_{u, v}: P^{\mathrm{op}} \rightarrow s \mathscr{S}$ et by

$$
H_{u, v}(S)=\mathfrak{C}(Z)\left(u, s_{1}\right) \times \mathfrak{C}(Z)\left(s_{1}, s_{2}\right) \times \cdots \times \mathfrak{C}(Z)\left(s_{n-1}, s_{n}\right) \times \mathfrak{C}(Z)\left(s_{n}, v\right),
$$

where by convention we have $H_{u, v}(\varnothing)=\mathfrak{C}(Z)(u, v)$.

A.2 Lemma Let $Z$ be an ordered simplicial set, and let $A \subseteq Z_{0}$ be a finite subset. Let $u, v \in Z$ and assume that every map $T \rightarrow Z_{u, v}$, where $T$ is a necklace, has at least one joint mapping into $A$. Then the composite map

$$
\underset{S \in P_{0}^{\mathrm{op}}}{\operatorname{hocolim}} H_{u, v}(S) \rightarrow \underset{S \in P_{0}^{\mathrm{op}}}{\operatorname{colim}} H_{u, v}(S) \rightarrow H_{u, v}(\varnothing)=\mathfrak{C}(Z)(u, v)
$$

is a Kan equivalence.

Proof Define a functor $F: P^{\text {op }} \rightarrow \mathscr{C}$ at by sending $S \in P$ to

$$
\left\{\left[T, T \hookrightarrow Z_{u, v}\right] \mid S \subseteq J_{T}\right\},
$$


the full subcategory of $\left(\mathcal{N e c} \downarrow Z_{u, v}\right)$ spanned by objects $T \stackrel{m}{\rightarrow} Z_{u, v}$ for which $m$ is an injection and $S \subseteq J_{T}$. Let us adopt the notation

$$
M_{S}(u, v)=\underset{T \in F(S)}{\operatorname{colim}} \mathfrak{C}(T)(\alpha, \omega) .
$$

This gives a functor $M_{(-)}(u, v): P^{\mathrm{op}} \rightarrow s \mathscr{S}$ et. Note that there is a natural map

$$
M_{\varnothing}(u, v) \longrightarrow \operatorname{colim}_{T \in\left(\mathcal{N e c} \downarrow S_{u, v}\right)} \mathfrak{C}(T)(\alpha, \omega) \cong \mathfrak{C}(Z)(u, v) .
$$

The first map is not a priori an isomorphism because in the definition of $F(\varnothing)$ we require that the map $T \rightarrow Z$ be an injection. However, using Lemma 4.12 (or Corollary 4.13 ) it follows at once that the map actually is an isomorphism.

From here the argument proceeds as follows. We will show:

(i) The natural map hocolim ${ }_{S \in P_{0}^{\text {op }}} M_{S}(u, v) \rightarrow \operatorname{colim}_{S \in P_{0}^{\text {op }}} M_{S}(u, v)$ is a Kan equivalence.

(ii) The map $\operatorname{colim}_{S \in P_{0}} M_{S}(u, v) \rightarrow M_{\varnothing}(u, v)$ is an isomorphism.

(iii) The functor $M_{(-)}(u, v)$ is naturally isomorphic to $H_{u, v}$.

These three items will clearly complete the proof.

For (i) we refer to [7, Section 13] and use the fact that $P_{0}^{\text {op }}$ has the structure of a directed Reedy category. Indeed, we can assign a degree function to $P$ that sends a set $S \subseteq A_{0}$ to the nonnegative integer $\left|A_{0}-S\right|$; all nonidentity morphisms in $P_{0}^{\text {op }}$ strictly increase this degree. By [7, Proposition 13.3] (but with $\mathscr{T}_{o p}$ replaced by $s \mathscr{S}$ et) it is enough to show that all the latching maps $L_{S}$ are cofibrations, where $L_{S}$ is the map

$$
L_{S}: \underset{S^{\prime} \supset S}{\operatorname{colim}} M_{S^{\prime}}(u, v) \rightarrow M_{S}(u, v)
$$

and the colimit is over sets $S^{\prime} \in P$ which strictly contain $S$. To see that $L_{S}$ is a cofibration, suppose that one has a triple $\left[T, T \hookrightarrow Z_{u, v}, t \in \mathfrak{C}(T)(\alpha, \omega)_{n}\right]$ representing an $n$-simplex of $M_{S^{\prime}}(u, v)$ and another triple $\left[T^{\prime}, T^{\prime} \hookrightarrow B_{u, v}, t^{\prime} \in \mathfrak{C}(U)(\alpha, \omega)_{n}\right]$ representing an $n$-simplex of $M_{S^{\prime \prime}}(u, v)$. If these become identical in $M_{S}(u, v)$ then it must be that they have the same flankification $\bar{T}=\bar{U}$ and $t=t^{\prime}$. Note that every joint of $T$ is a joint of $\bar{T}$, so the joints of $\bar{T}$ include both $S^{\prime}$ and $S^{\prime \prime}$. Because the joints of any necklace are linearly ordered, it follows that $S^{\prime} \cup S^{\prime \prime}$ is linearly ordered. Since $T \rightarrow \bar{T}$ is an injection, we may consider the triple $\left[\bar{T}, \bar{T} \hookrightarrow Z_{u, v}, t\right]$ as an $n$-simplex in $M_{S^{\prime} \cup S^{\prime \prime}}(u, v)$, which maps to the two original triples in the colimit; this proves injectivity. 
Assertion (ii) is the claim that the latching map $L_{\varnothing}: \operatorname{colim}_{S \in P_{0}^{\text {op }}} M_{S}(u, v) \rightarrow M_{\varnothing}(u, v)$ is an isomorphism. Injectivity was established above. For surjectivity, one needs to know that if $T$ is a necklace and $T \hookrightarrow Z_{u, v}$ is an inclusion, then $T$ must contain at least one vertex of $A$ as a joint. But this is precisely our assumption on $A$.

Finally, for (iii) fix some $S \in P_{0}$ and let $u=a_{0} \prec a_{1} \prec \ldots \prec a_{n} \prec a_{n+1}=v$ denote the complete set of elements of $S \cup\{u, v\}$. A necklace $T \hookrightarrow Z_{u, v}$ whose joints include the elements of $S$ can be split along the joints, and thus uniquely written as the wedge of necklaces $T_{i} \hookrightarrow Z_{a_{i}, a_{i+1}}$, one for each $0 \leq i \leq n$. Under this identification, one has

$$
\mathfrak{C}(T)(\alpha, \omega) \cong \mathfrak{C}\left(T_{0}\right)\left(\alpha_{0}, \omega_{0}\right) \times \cdots \times \mathfrak{C}\left(T_{n}\right)\left(\alpha_{n}, \omega_{n}\right) .
$$

Thus $F(S)$ is isomorphic to the category

$$
\left(\mathcal{N e c} \downarrow^{m} Z_{u, a_{1}}\right) \times\left(\mathcal{N e c} \downarrow^{m} Z_{a_{1}, a_{2}}\right) \times \cdots \times\left(\mathcal{N e c} \downarrow^{m} Z_{a_{n-1}, a_{n}}\right) \times\left(\mathcal{N e c} \downarrow^{m} Z_{a_{n}, v}\right),
$$

where $\left(\mathcal{N e c} \downarrow^{m} Z_{s, t}\right)$ denotes the category whose objects are $\left[T, T \rightarrow Z_{s, t}\right]$ where the map $T \rightarrow Z$ is a monomorphism.

Now, it is a general fact about colimits taken in the category of (simplicial) sets, that if $K_{i}$ is a category and $Q_{i}: K_{i} \rightarrow s$ Set is a functor, for each $i \in\{1, \ldots, n\}$, then there is an isomorphism of simplicial sets

$$
\underset{K_{1} \times \cdots \times K_{n}}{\operatorname{colim}}\left(Q_{1} \times \cdots \times Q_{n}\right) \stackrel{\cong}{\rightarrow}\left(\operatorname{colim}_{K_{1}} Q_{1}\right) \times \cdots \times\left(\underset{K_{n}}{\operatorname{colim}} Q_{n}\right) .
$$

Applying this in our case, we find that

$$
\begin{aligned}
M_{S}(u, v) & \cong \mathfrak{C}(Z)\left(u, a_{1}\right) \times \mathfrak{C}(Z)\left(a_{1}, a_{2}\right) \times \cdots \times \mathfrak{C}(Z)\left(a_{n-1}, a_{n}\right) \times \mathfrak{C}(Z)\left(a_{n}, v\right) \\
& =H_{u, v}(S) .
\end{aligned}
$$

This isomorphism is readily checked to be natural in $S$, so this proves (iii) and completes the argument.

A.3 Definition An ordered simplicial set $(X, \preceq)$ is called strongly ordered if, for all $a \preceq b$ in $X$, the mapping space $\mathfrak{C}(X)(a, b)$ is contractible.

Note that in any ordered simplicial set $X$ with $a, b \in X_{0}$, we have $a \preceq b$ if and only if $\mathfrak{C}(X)(a, b) \neq \varnothing$. Thus if $X$ is strongly ordered then its structure as a simplicial category, up to weak equivalence, is completely determined by the ordering on its vertices. We also point out that every necklace $T \in \mathcal{N} e c$ is strongly ordered by Corollary 3.10. 


\section{A.4 Lemma Suppose given a diagram}

$$
X \stackrel{f}{\leftarrow} A \stackrel{g}{\rightarrow} Y
$$

where $X, Y$, and $A$ are strongly ordered simplicial sets and both $f$ and $g$ are simple inclusions. Let $B=X \amalg_{A} Y$ and assume the following conditions hold:

(1) A has finitely many vertices.

(2) Given any $x \in X$, the set $A_{x \preceq}=\left\{a \in A \mid x \preceq_{B} a\right\}$ has an initial element (an element which is smaller than every other element).

(3) For any $y \in Y$ and $a \in A$, if $y \preceq_{Y} a$ then $y \in A$.

Then $B$ is strongly ordered.

Proof By Lemma 3.6, $B$ is an ordered simplicial set and the maps $X \hookrightarrow B$ and $Y \hookrightarrow B$ are simple inclusions. We must show that for $u, v \in B_{0}$ with $u \preceq v$, the mapping space $\mathfrak{C}(B)(u, v)$ is contractible. Suppose that $u$ and $v$ are both in $X$; then since $X \hookrightarrow B$ is simple, any necklace $T \rightarrow B_{u, v}$ must factor through $X$. It follows from Proposition 4.3 that $\mathfrak{C}(B)(u, v)=\mathfrak{C}(X)(u, v)$, which is contractible since $X$ is strongly ordered. The case $u, v \in Y$ is analogous. We claim we cannot have $u \in Y \backslash A$ and $v \in X \backslash A$. For if this is so and if $T \rightarrow B$ is a spine connecting $u$ to $v$, then there is a last vertex $j$ of $T$ that maps into $Y$. The 1 -simplex leaving that vertex then cannot belong entirely to $Y$, hence it belongs entirely to $X$. So $j$ is in both $X$ and $Y$, and hence it is in $A$. Then we have $u \preceq j$ and $j \in A$, which by assumption (3) implies $u \in A$, a contradiction.

It remains to show that if $u \in X, v \in Y \backslash A$, and $u \preceq_{B} v$ then $\mathfrak{C}(B)(u, v)$ is contractible. We claim that any map $T \rightarrow B_{u, v}$, where $T$ is a necklace, must send at least one joint of $T$ into $A$. To see this, recall that every simplex of $B$ either lies entirely in $X$ or entirely in $Y$. Since $v \notin X$, there is a last joint $j_{1}$ of $T$ which maps into $X$. If $C$ denotes the bead whose initial vertex is $j_{1}$, then the image of $C$ cannot lie entirely in $X$; so it lies entirely in $Y$, which means that $j_{1}$ belongs to both $X$ and $Y$-hence it belongs to $A$.

The preceding paragraph shows that we may apply Lemma A.2 to write

$$
\mathfrak{C}(B)(u, v) \simeq \underset{S \in P_{0}^{\mathrm{op}}}{\operatorname{hocolim}} H_{u, v}(S)
$$

where $P_{0}$ and $H_{u, v}(S)$ are defined prior to that lemma.

For each $S$ in $P_{0}$ we write $S=\left\{s_{1}, \ldots, s_{n}\right\}$ for $s_{i} \in A$ with $u \prec s_{1} \prec s_{2} \prec \cdots \prec s_{n} \prec v$. Then

$$
H_{u, v}(S)=\mathfrak{C}(B)\left(u, s_{1}\right) \times \mathfrak{C}(B)\left(s_{1}, s_{2}\right) \times \cdots \times \mathfrak{C}(B)\left(s_{n-1}, s_{n}\right) \times \mathfrak{C}(B)\left(s_{n}, v\right) .
$$


Because $X \hookrightarrow B$ is a simple inclusion, $\mathfrak{C}(X)\left(u, s_{1}\right) \rightarrow \mathfrak{C}(B)\left(u, s_{1}\right)$ is a Kan equivalence. For the same reason, the maps $\mathfrak{C}(X)\left(s_{i}, s_{i+1}\right) \rightarrow \mathfrak{C}(B)\left(s_{i}, s_{i+1}\right)$ and $\mathfrak{C}(Y)\left(s_{n}, v\right) \rightarrow \mathfrak{C}(B)\left(s_{n}, v\right)$ are Kan equivalences (in the latter case using that $Y \hookrightarrow B$ is also a simple inclusion). From the assumption that $X$ and $Y$ are strongly ordered we now have that all these mapping spaces are contractible. Hence $H_{u, v}(S)$ is contractible (this uses a special property of $s \mathscr{e}$, namely that a finite product of contractible spaces is contractible; this is an easy consequence of the fact that geometric realization preserves finite products).

At this point we know that the homotopy colimit on the right side of (A.5) is Kan equivalent to the nerve of $P_{0}^{\mathrm{op}}$. It thus suffices to prove that the nerve of $P_{0}$ (and hence

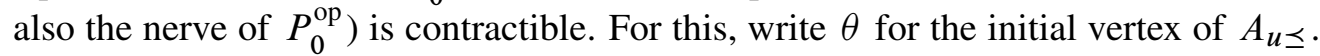
Define a functor $F: P_{0} \rightarrow P_{0}$ by $F(S)=S \cup\{\theta\}$; note that $S \cup\{\theta\}$ will be linearly ordered, so this makes sense. Clearly there is a natural transformation from the identity functor to $F$, and also from the constant $\{\theta\}$ functor to $F$. It readily follows that the identity map on $N P_{0}$ is homotopic to a constant map, hence $N P_{0}$ is contractible.

A.6 Proposition Let $T_{1}, \ldots, T_{m}$ be necklaces. Then their product $T_{1} \times \cdots \times T_{m}$ is a strongly ordered simplicial set.

Proof We begin with the case $P=\Delta^{n_{1}} \times \cdots \times \Delta^{n_{m}}$, where each necklace is a simplex, and show that $P$ is strongly ordered. It is ordered by Lemma 3.3, so choose vertices $a, b \in P_{0}$ with $a \preceq b$. If $T$ is a necklace, any map $T \rightarrow \Delta^{j}$ extends uniquely to a map $\Delta[T] \rightarrow \Delta^{j}$. It follows that any map $T \rightarrow P_{a, b}$ extends uniquely to $\Delta[T] \rightarrow P_{a, b}$. Consider the two functors

$$
f, g:\left(\mathcal{N e c} \downarrow P_{a, b}\right) \rightarrow\left(\mathcal{N e c} \downarrow P_{a, b}\right)
$$

where $f$ sends $[T, T \rightarrow P]$ to $[\Delta[T], \Delta[T] \rightarrow P]$ and $g$ is the constant functor sending everything to $\left[\Delta^{1}, x: \Delta^{1} \rightarrow P\right]$ where $x$ is the unique edge of $P$ connecting $a$ and $b$. Then clearly there are natural transformations id $\rightarrow f$ and $g \rightarrow f$, showing that the three maps id, $f$, and $g$ induce homotopic maps on the nerves. So the identity induces the null map, hence $\mathfrak{C}^{\text {nec }}(P)(a, b)=N\left(\mathcal{N e c} \downarrow P_{a, b}\right)$ is contractible. The result for $P$ now follows by Theorem 5.3.

For the general case, assume by induction that we know the result for all products of necklaces in which at most $k-1$ of them are not equal to beads. The case $k=1$ was handled by the previous paragraph. Consider a product

$$
Y=T_{1} \times \cdots \times T_{k} \times D
$$


where each $T_{i}$ is a necklace and $D$ is a product of beads. Write $T_{k}=B_{1} \vee B_{2} \vee \cdots \vee B_{r}$ where each $B_{i}$ is a bead, and let

$$
P_{j}=\left(T_{1} \times \cdots \times T_{k-1}\right) \times\left(B_{1} \vee \cdots \vee B_{j}\right) \times D .
$$

We know by induction that $P_{1}$ is strongly ordered, and we will prove by a second induction that the same is true for each $P_{j}$. So assume that $P_{j}$ is strongly ordered for some $1 \leq j<r$.

Let us denote $A=\left(T_{1} \times \cdots \times T_{k-1}\right) \times \Delta^{0} \times D$ and

$$
Q=\left(T_{1} \times \cdots \times T_{k-1}\right) \times B_{j+1} \times D .
$$

Then we have $P_{j+1}=P_{j} \amalg_{A} Q$, and we know that $P_{j}, A$, and $Q$ are strongly ordered. Note that the maps $A \rightarrow P_{j}$ and $A \rightarrow Q$ are simple inclusions: they are the products of the last-vertex-map $\Delta^{0} \rightarrow B_{1} \vee \cdots \vee B_{j}$ (resp. the initial-vertex-map $\Delta^{0} \rightarrow B_{j+1}$ ) with identity maps, and any map $\Delta^{0} \rightarrow V$, where $V$ is a necklace, is clearly simple. It is easy to check that hypotheses (1)-(3) of Lemma A.4 are satisfied, with $P_{j}$ playing the role of $X$ and $Q$ playing the role of $Y$. This finishes the proof.

Proof of Proposition 6.1 This follows immediately from Proposition A.6.

\section{A.7 The category $\mathfrak{C}\left(\Delta^{n}\right)$}

Our final goal is to give the proof of Lemma 2.5. We must construct isomorphisms

$$
\mathfrak{C}\left(\Delta^{n}\right)(i, j) \rightarrow N\left(P_{i, j}\right)
$$

for $n \in \mathbb{N}$ and $0 \leq i, j \leq n$, where $P_{i, j}$ is the poset of subsets of $\{i, i+1, \ldots, j\}$ containing $i$ and $j$. Moreover, we must verify that these isomorphisms are compatible with composition, thereby giving an isomorphism of simplicial categories $\mathfrak{C}\left(\Delta^{n}\right) \rightarrow N P$.

Proof of Lemma 2.5 The result is obvious when $n=0$, so we assume $n>0$. Let $m_{i}$ denote the unique map in $[n]$ from $i$ to $i+1$. One understands $F U([n])(i, j)$ as the set of free compositions of sequences of morphisms in $[n]$ which start at $i$ and end at $j$. Such free compositions are in one-to-one correspondence with the set of ways to "parenthesize" the word $m_{j} m_{j-1} \cdots m_{i+1}$ in such a way that each $m_{k}$ is contained in exactly one parenthesis. For example, when $n=3$ the maps in $F U([3])(0,3)$ are

$$
\left(m_{3} m_{2}\right)\left(m_{1}\right), \quad\left(m_{3}\right)\left(m_{2} m_{1}\right), \quad\left(m_{3} m_{2} m_{1}\right), \quad\left(m_{3}\right)\left(m_{2}\right)\left(m_{1}\right) .
$$

To such a parenthesization we associate the subset of $\{i, i+1, \ldots, j\}$ consisting of $i$ together with all indices that occur directly after a left parenthesis. The subsets of 
$\{0,1,2,3\}$ corresponding to the parenthesizations listed above, in order, are

$$
\{0,1,3\},\{0,2,3\}, \quad\{0,3\}, \quad\{0,1,2,3\} .
$$

It is easy to see that this gives a bijection between the maps in $F U([n])(i, j)$ and subsets of $\{i, i+1, \ldots, j\}$ containing $i$ and $j$.

Now let $X=(F U) \bullet([n])(i, j)$ and $Y=P_{i, j}$. For each $\ell \in \mathbb{N}$, we will provide an isomorphism $X_{\ell} \cong Y_{\ell}$, and these will be compatible with face and degeneracy maps. We have already done this when $\ell=0$.

For $\ell>0$ one has that $X_{\ell}$ is the set of free compositions of sequences of morphisms in $X_{\ell-1}$. It is readily seen that $X_{\ell}$ is in one-to-one correspondence with the set of ways to parenthesize the word $m_{j} m_{j-1} \ldots m_{i+1}$ in such a way that every element is contained in exactly $(\ell+1)$-many parentheses (and no closed parenthesis directly follows an open parenthesis). For example, the nine elements of $(F U)^{2}([3])(0,3)$ are

$$
\begin{aligned}
& \left(\left(m_{3} m_{2} m_{1}\right)\right), \quad\left(\left(m_{3} m_{2}\right)\right)\left(\left(m_{1}\right)\right), \quad\left(\left(m_{3}\right)\right)\left(\left(m_{2} m_{1}\right)\right), \quad\left(\left(m_{3}\right)\right)\left(\left(m_{2}\right)\right)\left(\left(m_{1}\right)\right), \\
& \left(\left(m_{3}\right)\left(m_{2}\right)\right)\left(\left(m_{1}\right)\right), \quad\left(\left(m_{1}\right)\left(m_{2}\right)\left(m_{3}\right)\right), \quad\left(\left(m_{3}\right)\right)\left(\left(m_{2}\right)\left(m_{1}\right)\right), \quad\left(\left(m_{3}\right)\left(m_{2} m_{1}\right)\right), \\
& \left(\left(m_{3} m_{2}\right)\left(m_{1}\right)\right) \text {. }
\end{aligned}
$$

Given such a parenthesized sequence, one can rank the parentheses by "interiority" (so that interior parentheses have higher rank). The face and degeneracy maps on $X$ are given by deleting or repeating all the parentheses of a fixed rank. Under this description, a vertex in an $\ell$-simplex of $X$ is given by choosing a rank and then ignoring all parentheses except those of that rank. Such a vertex determines a subset of $\{i, i+1, \ldots, j\}$ containing $i$ and $j$, as in the first paragraph of this proof. And given two ranks, the subset of $\{i+1, \ldots, j\}$ corresponding to the higher rank will contain the subset corresponding to the lower rank (due to the nested nature of the parentheses).

One can check that an $\ell$-simplex in $X$ is determined by its set of vertices, and so we can identify $X_{\ell}$ with the set of sequences $S_{0} \subseteq S_{1} \subseteq \cdots \subseteq S_{\ell} \subseteq\{i, i+1, \ldots, j\}$ containing $i$ and $j$. This is precisely the set of $\ell$-simplices of $Y$, so we have our isomorphism $X_{l} \cong Y_{l}$. It is clearly compatible with face and degeneracy maps.

Finally, we point out that the composition in the category $\mathfrak{C}\left(\Delta^{n}\right)$ corresponds to concatenation of parenthesized words. From this one readily checks that the above isomorphisms give a map (thus, an isomorphism) of categories $\mathfrak{C}\left(\Delta^{n}\right) \rightarrow N P$.

\section{References}

[1] M Artin, B Mazur, Etale homotopy, Lecture Notes in Math. 100, Springer, Berlin (1969) MR0245577 
[2] H J Baues, Geometry of loop spaces and the cobar construction, Mem. Amer. Math. Soc. 25, no. 230 (1980) MR567799

[3] J E Bergner, A model category structure on the category of simplicial categories, Trans. Amer. Math. Soc. 359 (2007) 2043-2058 MR2276611

[4] A K Bousfield, D M Kan, Homotopy limits, completions and localizations, Lecture Notes in Math. 304, Springer, Berlin (1972) MR0365573

[5] J-M Cordier, Sur la notion de diagramme homotopiquement cohérent, from: "Third Colloquium on Categories, Part VI (Amiens, 1980)", Cahiers Topologie Géom. Différentielle 23 (1982) 93-112 MR648798

[6] J-M Cordier, T Porter, Vogt's theorem on categories of homotopy coherent diagrams, Math. Proc. Cambridge Philos. Soc. 100 (1986) 65-90 MR838654

[7] D Dugger, A primer on homotopy colimits, Preprint (2008) Available at http:// math. uoregon. edu/ ddugger/hocolim.pdf

[8] D Dugger, D I Spivak, Mapping spaces in quasi-categories, Alg. Geom. Topol. 11 (2011) 225-261

[9] W G Dwyer, D M Kan, Function complexes in homotopical algebra, Topology 19 (1980) 427-440 MR584566

[10] PS Hirschhorn, Model categories and their localizations, Math. Surveys and Monogr. 99, Amer. Math. Soc. (2003) MR1944041

[11] A Joyal, Quasi-categories and Kan complexes, J. Pure Appl. Algebra 175 (2002) 207222 MR1935979 Special volume celebrating the 70-th birthday of Professor Max Kelly

[12] A Joyal, The theory of quasi-categories, Preprint (2008)

[13] J Lurie, Higher topos theory, Annals of Math. Studies 170, Princeton Univ. Press (2009) MR2522659

[14] S Mac Lane, Categories for the working mathematician, second edition, Graduate Texts in Math. 5, Springer, New York (1998) MR1712872

[15] D Quillen, Higher algebraic $K$-theory. I, from: "Algebraic $K$-theory, I: Higher $K$ theories (Proc. Conf., Battelle Memorial Inst., Seattle, Wash., 1972)”, (H Bass, editor), Lecture Notes in Math. 341, Springer, Berlin (1973) 85-147 MR0338129

Department of Mathematics, University of Oregon Eugene OR 97403, USA

Department of Mathematics, Massachusetts Institute of Technology Building 2, Room 236, 77 Massachusetts Avenue, Cambridge MA 02139-4307, USA ddugger@uoregon.edu, dspivak@math.mit.edu http://pages.uoregon.edu/ddugger/, http://math.mit.edu/ dspivak/

Received: 22 December 2009 Revised: 9 July 2010 